\title{
Model Predictive Active Power Control for Optimal Structural Load Equalization in Waked Wind Farms
}

\author{
Mehdi Vali, Vlaho Petrović, Lucy Y. Pao, and Martin Kühn
}

\begin{abstract}
In this paper, we propose a model predictive active power control (APC) enhanced by the optimal coordination of the structural loadings of wind turbines operating with fully developed wind farm flows that have extensive interactions with the atmospheric boundary layer. In general, the APC problem, that is, distributing a wind farm power reference among the operating wind turbines, does not have a unique solution; this fact can be exploited for structural load alleviation of the individual wind turbines. Therefore, we formulated a constrained optimization problem to simultaneously minimize the wind farm power reference tracking errors and the structural load deviations of the wind turbines from their mean value. The wind power plant is represented by a dynamic 3D large-eddy simulation model, whereas the predictive controller employs a simplified, computationally inexpensive model to predict the dynamic power and load responses of the turbines that experience turbulent wind farm flows and wakes. An adjoint approach is an efficient tool used to iteratively compute the gradient of the formulated parameter-varying optimal control problem over a finite prediction horizon. We have discussed the applicability, key features, and computational complexity of the controller by using a wind farm example consisting of $3 \times 4$ turbines with different wake interactions for each row. The performance of the proposed adjoint-based model predictive control for APC was evaluated by measuring power reference tracking errors and the corresponding damage equivalent fatigue loads of the wind turbine towers; we compared our proposed control design with recently published proportionalintegral-based APC approaches.
\end{abstract}

Index Terms-Optimal control, Adjoint approach, Wind farm control, Wake effects, Active power control, Structural load reduction.

\section{INTRODUCTION}

$\mathbf{T}$ HE control of turbines in a wind farm is challenging because of the aerodynamic interactions through their wakes. The main characteristics of a wake are reduced wind

This work was partly funded by the Ministry for Science and Culture of Lower Saxony through the funding initiative "Niedersächsisches Vorab" (project "ventus efficiens") and by the Federal Ministry for Economic Affairs and Energy according to a resolution by the German Federal Parliament ("WIMS-Cluster" 0324005). Support from the HanseWissenschaftskolleg in Delmenhorst, Germany and from a Palmer Endowed chair at the University of Colorado Boulder is also gratefully acknowledged.

M. Vali, V. Petrović, and M. Kühn are with the ForWind-Center for Wind Energy Research, Institute of Physics, Carl von Ossietzky University of Oldenburg, Küpkersweg 70, 26129 Oldenburg, Germany (e-mail: mehdi.vali@uol.de; vlaho.petrovic@uol.de; martin.kuehn@uol.de)

L. Y. Pao is with the Department of Electrical, Computer \& Energy Engineering, University of Colorado, Boulder, CO 80309 USA (e-mail: pao@Colorado.EDU). speed and increased turbulence; the former diminishes the total power production of the farm, and the latter leads to a high dynamic loading on the downstream turbines [1]. In a wind farm, wakes often merge with one another, which creates multiple wakes. The increased turbulence intensity inside the wakes, wake meandering, and partial overlapping of the rotors of downwind turbines by wakes cause the turbines inside the wind farms to be prone to high structural fatigue loads. The wake interactions also depend strongly on the variations in the wind direction, turbulence, atmospheric stratification, surface roughness, local terrain, and turbine layout in the wind farm [2], [3].

The main objectives of wind farm control can be categorized as follows [4]:

1) Power maximization, e.g., by minimizing the wakeinduced power losses [5], [6], [7],

2) Active power control (APC), wherein the total wind farm power production follows a power reference signal provided by the transmission system operator (TSO) [8], [9],

3) Aggregated structural load alleviation, e.g., by coordinating the power distributions among the individual wind turbines or by mitigating the wake-induced dynamic loads [10], [11], [12],

4) Voltage and frequency control of wind farms for connecting to the grid [13], [14].

The focus of this paper is on the second and third abovementioned aims. However, first it is useful to explain the current practice and ongoing research concerning wind turbine control in wind farms with respect to power extraction/maximization. In this application a shift of the operational objective from turbine to the wind farm level is taking place which is important for the later discussed active power control as well.

Traditionally, concerning power maximization, each turbine employs greedy control, that is, it optimizes its own performance by neglecting the impact on downstream turbines through the wake. By changing operating points of the upwind turbines, it is possible to influence the wakes and the performance of the downwind turbines operating inside the wakes. Reducing the power capture of an upwind turbine increases the energy content of the wake reaching a downstream turbine. A structural load balance might also be achieved by transferring loads to the upwind turbines 
to mitigate the wake-induced structural loadings on the downstream turbines [4]. Unlike greedy control, system optimization aims to find control solutions to minimize the undesirable effects of wind farm wakes and improve the overall wind farm performance. Two commonly proposed approaches for wake control of wind farms are induction control [5], [6] and wake-steering control [7].

Structural load alleviation in wind farms is generally not addressed as an isolated control objective since it is considered as an additional desire during operation for power maximisation, which is still by far the most frequent operational mode, or during grid related operational modes. In the following, an overview of the state-of-the-art in APC and approaches for structural load alleviation are given.

In active power control (APC), the wind power plant responds to the grid requirements by controlling the total power production. APC has been investigated for balancing the power supply with demands on both the wind turbine [15], [16] and wind farm levels [8], [9]. Many studies [13] have employed multiple decoupled wind turbine simulations wherein wind inputs were uncorrelated. The first investigation on challenges posed by the aerodynamic interactions of the turbines for APC of wind farms was done in [8]. In [8], the wind turbines were coordinated through an open-loop supervisory controller that evenly derated the power production of the individual wind turbines to address the total wind farm power demand from the TSO. However, the local wake and turbulence effects reduced the available wind power in reserve; therefore, some downwind turbines failed to follow their set-points. In [9], van Wingerden et al. have extended the investigation by designing a wind farm feedback controller that could appropriately adjust the turbine set-points to compensate for the accumulated wake-induced power tracking errors. Vali et al. [17] exploited the non-uniqueness of the APC solution with respect to the power/load distributions for actively adjusting the wind turbine power set-points for wake-induced structural load alleviation while maintaining the tracking performance of the wind farm power references. Although the proportional-integral (PI)-based APC with a coordinated load distribution (CLD) law [17] only requires a simple control architecture and practical sensory systems, it is not easy to find an optimal control solution and properly deal with practical constraints.

Recently, several studies have developed model predictive control (MPC) for both power maximization [18], [19], [20] and APC [12], [21], [22], [23] of waked wind farms. A wide range of predictive wind farm models ranging from highfidelity to static low-fidelity models have been investigated for a reliable and practical MPC design of wind farms in the presence of wake effects.

To optimize the overall wind farm performance, [18], [19] and [24] used a fully 3D large-eddy simulation (LES) model. LES models of wind farms are computationally expensive and are typically used for detailed investigations of the interactions of turbulent flows, wakes, and wind turbines with the atmospheric boundary layer. Ideally, a controloriented model should capture the relevant dominant flow dynamics in a computationally inexpensive manner [25]; this is the main motivation of studying medium-fidelity dynamic models of wind farms [26], [27], [28], [29].

In [20] and [22], we developed an adjoint-based MPC (hereinafter AMPC) scheme which relies on a 2D mediumfidelity wind farm model (i.e., WFSim [29]) for power maximization and optimal APC of waked wind farms; it takes dynamic wake interactions into account. WFSim is a controloriented dynamic wind farm flow model characterized by reasonably low computational costs, and it is modeled by using the 2D Navier Stokes equations constrained by the continuity equation. An important feature of WFSim is the sparsity in the system matrices, which improves the computational efficiency of the prediction model [30]. However, the order of the prediction model can easily grow depending on the meshing resolution of the wind farm domain [29]. Therefore, we implemented an adjoint approach to efficiently compute the gradient of the MPC performance index with the same computational effort as the prediction model; the gradient was computed independently of the order of the model and the optimization problem.

From the literature, it is evident that a reliable controloriented wind farm model plays a key role in the reliable performance and implementation of the MPC scheme. It has been shown that adequate MPC formulations and parameterizations can significantly reduce the computational complexity [20]. However, the overall complexity of such a control system, including a suitable mathematical model and measurement system [31], is still an open research topic, particularly for large wind farms with up to hundred or more wind turbines. Distributed MPC has recently received considerable attention for reducing the computational burden of the model predictive APC [32].

Shapiro et al. [21] formulated a model predictive APC that relied on a one-dimensional static low-fidelity wake model. The same MPC problem was formulated in [12], [23] using only wind turbine dynamic responses that neglected their wake interactions in the controller design. Further, [9] showed that a simple PI-based feedback from the total wind farm power was enough to guarantee a satisfactory power reference tracking performance. The PI-based APC has recently been validated using wind tunnel experiments as well [33]. However, the literature does not properly address the distribution of power references among wind turbines.

A $H_{2}$-optimal control based on a 2D wake model [11], a linear quadratic gaussian (LQG) control [34], and a MPC [12], [35] were formulated for simultaneous power reference tracking and load control, although the performance of the controllers was assessed by using simplified engineering wind farm simulation tools. To obtain a more comprehensive understanding of the underlying physics and how control techniques can influence dynamic wind farm flows, computational fluid dynamics (CFD) models tuned with experimental data have been typically employed [36]. 
TABLE I

ACTIVE POWER CONTROL STATE-OF-THE-ART METHODS WITH STRUCTURAL LOAD CONTROL FROM DIFFERENT CONTROL ARCHITECTURES AND VALIDATION APPROACHES. VALIDATION WITH ENGINEERING WIND FARM SIMULATION MODELS ARE IN RED; HIGH-FIDELITY LES MODELS ARE IN GREEN; AND WIND TUNNEL EXPERIMENTS ARE IN BLUE.

\begin{tabular}{|c|c|c|c|c|}
\hline $\begin{array}{l}\text { Control } \\
\text { architecture }\end{array}$ & $\begin{array}{l}\text { Control-oriented } \\
\text { wake model }\end{array}$ & $\begin{array}{l}\text { Reference APC } \\
\text { (no load control) }\end{array}$ & $\begin{array}{l}\text { APC with load } \\
\text { reduction }\end{array}$ & $\begin{array}{l}\text { APC with load } \\
\text { equalization (CLD) }\end{array}$ \\
\hline Model-free & N.A. & $\begin{array}{l}\text { Open-loop [8] } \\
\text { PI-based [9], [13], [33] }\end{array}$ & - & PI-based [17] \\
\hline $\begin{array}{l}\text { Model-based, } \\
\text { relies on wind turbine } \\
\text { model with different } \\
\text { wake models }\end{array}$ & $\begin{array}{l}\text { 3D wake model } \\
\text { 2D wake model } \\
\text { 1D wake model } \\
\text { No wake model }\end{array}$ & $\begin{array}{l}\text { AMPC [24] } \\
\text { AMPC [22] } \\
\text { distributed MPC [32] } \\
\text { AMPC [21] } \\
\text { - }\end{array}$ & $\begin{array}{l}- \\
H_{2} \text {-optimal control [11] } \\
- \\
\text { MPC [12], [23], [35] } \\
\text { LQG control [34] }\end{array}$ & $\begin{array}{l}- \\
- \\
- \\
\text { AMPC [current study] }\end{array}$ \\
\hline
\end{tabular}

These models allow resolving time-varying turbulent flows for better characterization of the wake physics (e.g., the shape of the velocity deficit inside the wake, wake-added turbulence, wake meandering, different atmospheric stabilities, and multiple wakes).

Table I summarizes the aforementioned state-of-the-art method for the APC of wind farms. The four criteria considered are control architectures, the employed controloriented wake model characteristics, APC objectives, and the validation approaches. The APC approaches with load reduction in [12], [23], [34], [35] are formulated to locally penalize the variations in the power production of the individual wind turbines and thus indirectly reduce their structural fatigue loadings. However, the coordinated load distribution (CLD) law [17] equalizes the structural loading of wind turbines that operate in wind farm flows with different wake and non-wake conditions using direct measurements obtained from the load sensors. One important note is that the load equalization concept originates from the irregular load pattern of wind farms [37]. Load equalization is possible due to the flexibility of the APC solution domain to transfer wake-induced loading from downstream turbines to the upwind ones without deteriorating the wind farm power reference tracking performance. It would be beneficial for a wind farm to have all turbines similarly loaded/worn. Another positive aspect is that the load equalization approach tends to avoid control saturation of downwind turbines and increases their controllability for rejecting wake-induced dynamic loadings [17]. To evaluate the reliability of the results, the validation method of each investigation is indicated with different colors in Table I. The red, green, and blue colors are associated with validations using low-fidelity engineering wind farm simulation tools, high-fidelity CFD models, and wind tunnel experiments, respectively. Note that field tests and current industrial practice are limited to open-loop approaches with or without only simplified wake interaction models.

The main contribution of this paper is to design a new model predictive APC with further reduced computational complexity to simultaneously minimize the wake-induced power tracking errors and equalize the structural fatigue loads of the individual wind turbines within a fully developed wind farm boundary layer. The proposed MPC scheme exploits the non-uniqueness of the APC solution for optimal power distribution among the individual wind turbines, while their total power production follows a power reference. A constrained optimization problem was formulated to address the desired multi-objective control problem and to handle practical constraints. Contrary to the AMPC design in [20] and [22], the new predictive control design relies on a much more simplified wind farm model, which is composed of the decoupled individual wind turbine models. The spatial propagation of the wake model was neglected in the AMPC architecture presented in this paper. The effect of dynamic wakes was treated as a disturbance and were captured through online adaptation of the controller model using direct measurements from individual wind turbines. The parameter-varying nature of the formulated optimization problem benefits from the adjoint method for the online computation of the gradient. The new AMPC has lower computational complexity and a simpler measurement system than [20], which makes it suitable for real-time control of waked wind farms. LESs were used for detailed testing of the applicability of the control design under dynamic wakes and turbulent conditions. The performance of the AMPC-based APC for structural load equalization and its computational complexity have been compared with recently designed PIbased APC approaches in [9] and [17].

The remainder of this paper is organized as follows. In Section II, we briefly present the employed wind power plant model. The main focus of Section III is the structure of the proposed MPC-based APC of wind farms. Then, the design characteristics and the performance of the controller is discussed through comparative LES studies in Section IV. Finally, the strengths and weaknesses of the proposed approach are outlined in Section V as conclusions. 


\section{WIND FARM REPRESENTATION}

For wind farm control, a minimum set of measurements is required at the turbine level. Many modern wind turbines measure structural loads, e.g., on the blades or the tower. As mentioned before, the objective of our proposed control approach is to minimize the computational complexity by avoiding an expensive wind farm prediction model and sophisticated sensors. To demonstrate this, we use a representation of the wind power plant including its wake interaction that requires only the following essential sensors: power output, nacelle wind speed, turbine load quantifier, e.g., tower base fore-aft bending moment. The following subsection describes the large-eddy simulation (LES) model employed in our study for this aim. In principle, the low requirements on the measurement system would allow an implementation of the proposed controller on wind tunnel models as well as on real wind farms.

\section{A. Large-eddy simulation model of the wind farm}

The LES approach has the capability of resolving the unsteady nature of the wake and turbulent flows within a wind farm [38], [39]. For wind farm control applications, the aerodynamics of wind turbine wakes is typically described using actuator disc theory [40]. Fig. 1 schematically depicts the two main components of the employed wind farm simulation model. The employed LES model is coupled with the $N_{t}$ wind turbine models to establish the investigated wind farm case study, where $N_{t}$ is the number of turbines. The amount of energy capture of a wind turbine and the induced velocity variation are regulated using the axial induction factor $a$, that is, the ratio of the reduced wind velocity $U_{d}$ at the rotor plane to the undisturbed wind speed $U_{0}$ far ahead of the rotor. The $i^{\text {th }}$ wind turbine applies its axial thrust force $F_{a, i}$ into the incoming flow; its wake propagates downstream and interacts with the atmospheric boundary layer and downstream turbines. The wake-added turbulence mixes the low-velocity flow inside the wake with the high-velocity flow outside the wake, which re-energizes the wake and accelerates the wake recovery. The background atmospheric boundary layer, especially in the first rows, has a huge impact on the characteristics of wake recovery.
In this study, we use the PArallelized LES Model (hereinafter PALM) [41] coupled with the actuator disc model (ADM) of a wind turbine [42]. PALM is an open source and advanced meteorological model for simulating atmospheric and oceanic flows, developed as a turbulence-resolving LES code and optimally designed to be executed on massively parallel computer architectures. PALM uses the Schumann volume averaging approach [43] and uses central differences to discretize the non-hydrostatic and incompressible Boussinesq approximation of the three-dimensional Navier-Stokes equations on a structured Cartesian grid. The spatial filtering causes the LES approach to only simulate the large eddies of turbulence. Hence, the dynamic effect of small turbulent scales is considered with a so-called subgrid model. PALM is also capable of simulating the effect of the Coriolis forces. More information about PALM is available in [41]. Much more detailed wind turbine models with more realistic nearwake structures, such as the ADM with rotation (ADM-R) and actuator line model (ALM), have also been implemented in PALM. However, ADM is computationally efficient and provides a good approximation of the far-wake structure, which makes it suitable for this study.

\section{B. Wind turbine model}

The individual wind turbines are parameterized with an ADM to exert a thrust force on the incoming flow and extract a certain amount of energy from the incoming wind. The wind turbines are modeled in PALM using a third-order nonlinear state-space representation as follows:

$$
\begin{aligned}
\tau \dot{P}+P & =P_{a}\left(a, U_{0}, \dot{x}_{T}\right), \\
m_{T_{e}} \ddot{x}_{T}+c_{T} \dot{x}_{T}+k_{T} x_{T} & =F_{a}\left(a, U_{0}, \dot{x}_{T}\right) .
\end{aligned}
$$

Equation (1) represents the electrical power response of a wind turbine $P$ to the aerodynamic power $P_{a}$ with the aerodynamic time constant $\tau$, which can be associated with the drive-train dynamics [17]. The aerodynamic power is a function of the effective wind speed $U_{0}$, i.e., the inflow wind speed not affected by the rotor induction, the induction factor $a$, and the tower top velocity $\dot{x}_{T}$. Equation (2) describes the first tower fore-aft dynamic mode with the tower top
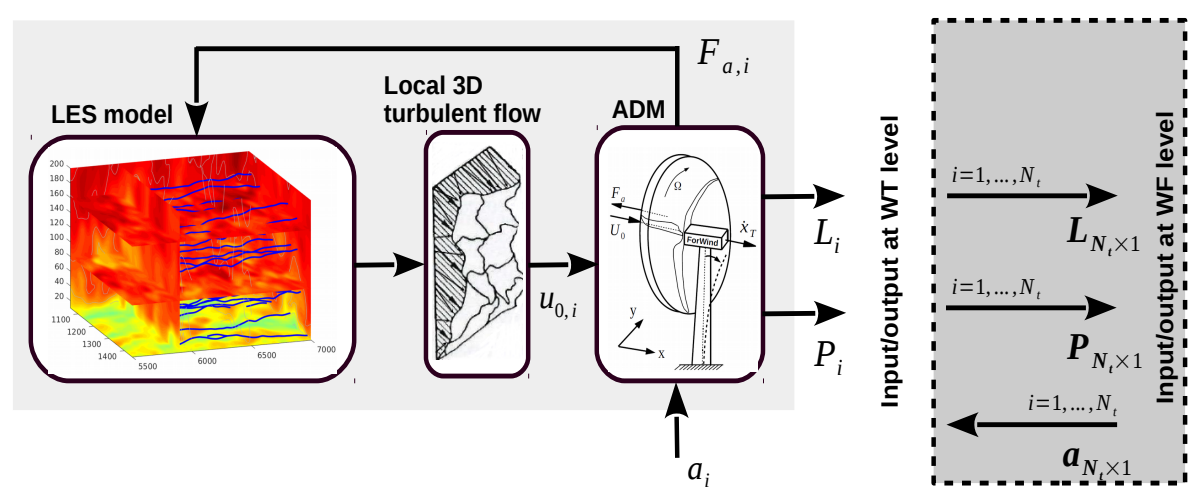

Fig. 1. Schematic illustration of the wind farm simulation model. WF is the wind farm, and WT is the wind turbine. The dark grey block represents the input/outputs at the WF level and the input/outputs collected at the WT level. 
fore-aft displacement $x_{T}$, aerodynamic thrust force $F_{a}$, towerequivalent modal mass $m_{T_{e}}$, structural damping $c_{T}$, and bending stiffness $k_{T}$ [44]. The tower top fore-aft displacement $x_{T}$ is considered positive in the downwind direction.

We calculate the aerodynamic thrust force for a single turbine by using the employed ADM in the PALM simulation code as follows [45]:

$$
F_{a}=\frac{1}{2} \rho A_{d} U_{\mathrm{rel}}^{2} C_{T}(a)
$$

where $\rho$ is the air density, and $A_{d}$ is the swept area of the rotor plane; the thrust coefficient $C_{T}$ is a function of the induction factor $a$, i.e., the wind turbine control input, as:

$$
C_{T}(a)=4 a(1-a) .
$$

The axial induction factor is limited to its value for the maximum (greedy) power extraction [45] (i.e., $a \leq \frac{1}{3}$ ) to avoid high wake losses and regions where the thrust coefficient (4) does not hold. It has been shown experimentally that the assumption of an ideal wind turbine is not valid for high axial induction factors due to wake separation and a high jump between wind speeds far upstream and downstream of the wind turbine [46]. The relative wind speed $U_{\text {rel }}$ is defined as a superposition of the effective wind speed $U_{0}$ and the structural tower top velocity as follows [44]:

$$
U_{\text {rel }}=U_{0}-\dot{x}_{T}
$$

This is done to model the rotor fore-aft motion and the induced aerodynamic damping of the tower fore-aft mode.

By resolving equation (5) and considering the induction effect of a rotor disc, the effective wind speed $U_{0}$ is ap- proximated from the measurable axial disc-averaged wind velocity $U_{d}$ from PALM and the tower top velocity $\dot{x}_{T}$ as

$$
\hat{U}_{0}=\frac{U_{d}}{1-a}+\dot{x}_{T},
$$

which enables us to model the applied aerodynamic thrust force (3) acting in the negative direction on the flow [42]. Note that it is assumed that the real induction factor is instantaneously following the commanded value $a$. Then, the aerodynamic power of an individual wind turbine is calculated as follows:

$$
P_{a}=F_{a} U_{d} .
$$

The tower base fore-aft bending moment of an individual wind turbine is approximated as follows [44]:

$$
M_{T}=h_{H}\left(c_{T} \dot{x}_{T}+k_{T} x_{T}\right),
$$

where $h_{H}$ is the hub height. Similar to [17], the tower base fore-aft bending moment is used as a representative load indicator to mitigate the wake-induced global load variations for different wind turbines inside the wind farm rather than to determine the actual fatigue load damage of the tower or any other component.

It is important to note that the ADM integrates the turbulence-induced loads over the entire swept area and neglects important dynamic load effects, for example, the rotational sampling of the partial gusts and partial wakes. If a more realistic rotor model (e.g., the ALM with individual blades) is applied, the modeling of the structural dynamics and the load effect of the turbine should be improved as well. In addition, power de-rating control is governed by the induction control of the individual wind turbines. A more comprehensive wind turbine control system would consider

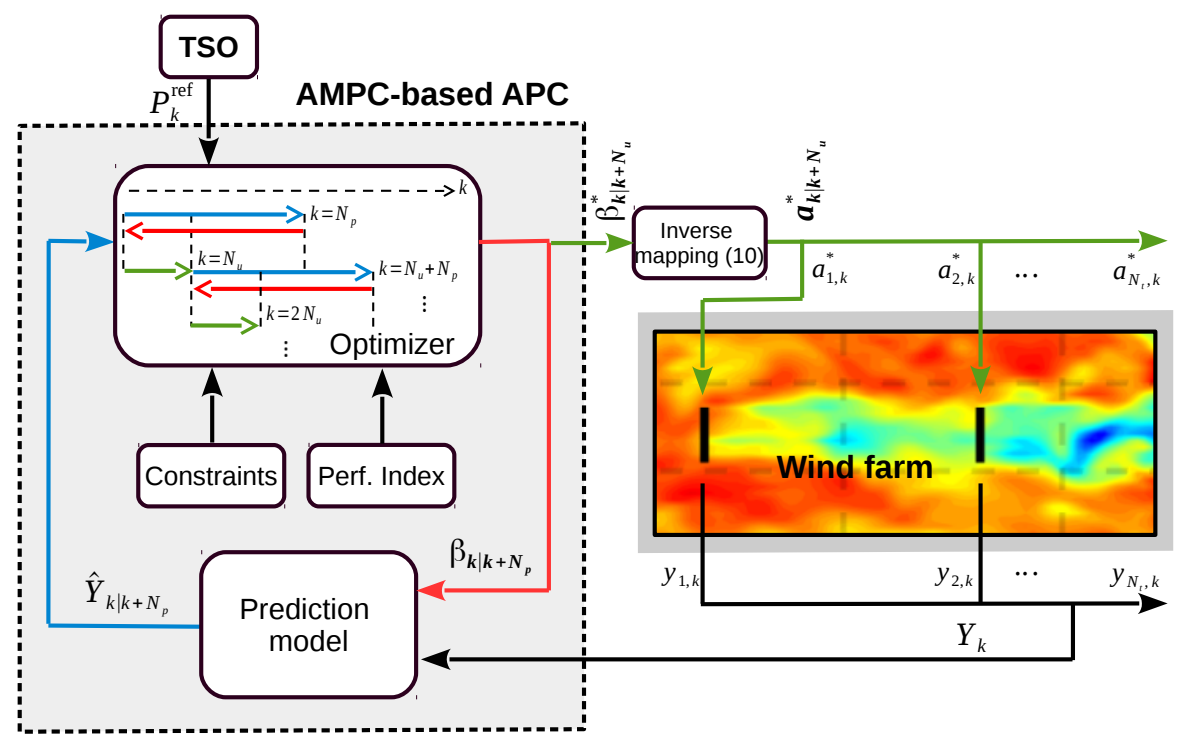

Fig. 2. Schematic illustration of the AMPC-based APC of wind farms with equalized structural load coordination. The dashed box contains the main components of the AMPC. The virtual induction factors $\boldsymbol{\beta}_{k}^{\star}$ are optimally adjusted in such a way that the total wind farm power follows the power reference signal $P_{k}^{\text {ref }}$, demanded from the TSO at each time instant $k$. The optimal induction factors $\boldsymbol{a}_{k}^{\star}$ are then applied by using the inverse mapping of (10). The measurement signal $\boldsymbol{Y}_{k}$ stacks all the power $\boldsymbol{P}_{k}$ and structural load $\boldsymbol{L}_{k}$ values of the individual wind turbines, which are the outputs from the plant model (dark grey blocks in Fig. 1 and 2). The wind power plant was simulated using a LES model with relatively realistic three-dimensional wind farm flows (see section II). 
the actual torque and pitch controllers. The authors of [16] have provided additional details on the practical aspects of implementing the torque and pitch control for power derating and have studied the effects of these controllers on the wind turbine structural loads.

\section{MOdEL PREDICTIVE ACTIVE POWER CONTROL}

We propose a model predictive APC framework to optimally coordinate the power production of the individual wind turbines for equalizing the structural fatigue loadings in a waked wind farm. Fig. 2 schematically depicts the control architecture of the proposed MPC. The dashed box contains the main components of the developed AMPC for the APC of waked wind farms. The proposed MPC relies on a simplified model for predicting the optimal input/output trajectories (i.e., the virtual induction factors $\boldsymbol{\beta}_{k} \in \mathbb{R}^{N_{t}}$ and the individual wind turbine power/load $\boldsymbol{Y}_{k}=\left[\begin{array}{ll}\boldsymbol{P}_{k} & \boldsymbol{L}_{k}\end{array}\right]^{T} \in \mathbb{R}^{2 N_{t}}$ ) over the prediction horizon $N_{p}$. The optimizer benefits from an adjoint method as an effective tool to iteratively compute the gradient of the formulated optimization problem. The parameter-varying system matrices and the performance index elements are linearized and stored online over the prediction horizon $N_{p}$ (see blue arrows). The adjoint variable associates the variations of the prediction model with the performance index, and is used for the online calculation of the gradient backward in time (see red arrows). On the basis of the receding horizon principle, we applied the first part of the optimal solution to the wind power plant over the control horizon $N_{u}<N_{p}$ (see green arrows), and the whole procedure was repeated with new measurements, providing feedback into the optimal control problem.

\section{A. Prediction model}

This section presents the fundamentals of the employed prediction model in the proposed MPC scheme. It has been shown that a satisfactory wind farm power reference tracking performance [9] and a simultaneous structural load coordination [17] can be achieved using classical control theories. Boersma et al. [23] formulated an MPC scheme for power reference tracking using only wind turbine models that overlooked their aerodynamic interactions via wakes. In this paper, the MPC aims to optimize the power/load distribution trajectories among individual wind turbines while their total production follows the TSO wind farm power command. Wind turbines can change their power outputs and react to wake disturbances much faster than the wake propagations. Therefore, the MPC preview optimizations can be shorter than the wake propagation time from one turbine toward its downstream ones. This means that the wake propagation model is not needed due to its longer time scale, compared with the one needed for APC. Hence, similar to [23] and in contrast to [20], the spatial propagation of the wake toward downstream is omitted here when finding an APC solution. Consequently, the wind farm prediction model is composed of uncoupled subsystems, which represents the dynamic power and structural load of individual wind turbines that experience turbulent wind farm flows and wakes at each time instant $k$.

Substituting (4)-(6) into (3) enables one to rewrite the thrust force of the $i^{\text {th }}$ wind turbine at time instant $k$ as:

$$
F_{a_{i}, k}=2 \rho A_{d} U_{d_{i}, k}^{2} \beta_{i, k}
$$

with a linear representation with respect to the virtual control input $\beta_{i, k}$. The following mapping was applied to the axial induction factor $a_{i, k}$ to introduce the new wind turbine control input $\beta_{i, k}$ [30], the so-called virtual induction factor:

$$
\beta_{i, k}=\frac{a_{i, k}}{1-a_{i, k}}
$$

Then, a discretized multi-input multi-output state-space model was constructed by discretizing (1) and (8) as follows:

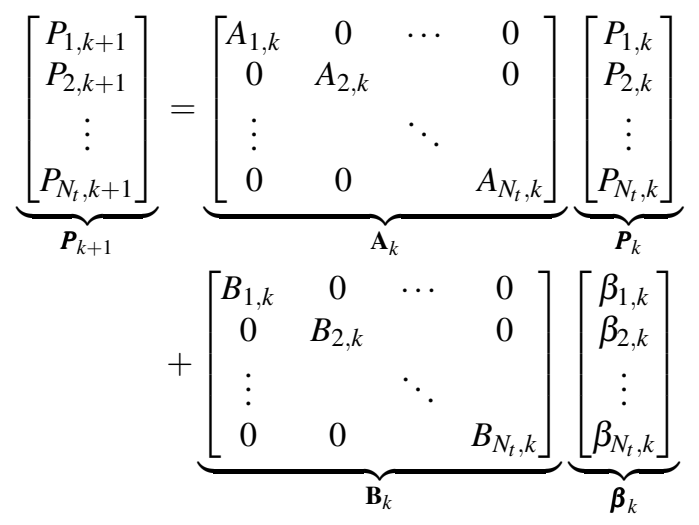

$$
\underbrace{\left[\begin{array}{c}
M_{1, k} \\
M_{2, k} \\
\vdots \\
M_{N_{t}, k}
\end{array}\right]}_{\boldsymbol{L}_{\boldsymbol{k}}}=\underbrace{\left[\begin{array}{cccc}
D_{1, k} & 0 & \cdots & 0 \\
0 & D_{2, k} & & 0 \\
\vdots & & \ddots & \\
0 & 0 & & D_{N_{t}, k}
\end{array}\right]}_{\mathbf{D}_{k}} \underbrace{\left[\begin{array}{c}
\beta_{1, k} \\
\beta_{2, k} \\
\vdots \\
\beta_{N_{t}, k}
\end{array}\right]}_{\boldsymbol{\beta}_{k}}
$$

for predicting the power production $\boldsymbol{P}_{k} \in \mathbb{R}^{N_{t}}$ and the structural loading $\boldsymbol{L}_{k} \in \mathbb{R}^{N_{t}}$ of the waked wind farm consisting of $N_{t}$ wind turbines. Similar to [9], [17], and [23], possible dynamic interactions between the individual turbines have been ignored in the prediction model, which results in diagonal system matrices. Furthermore, to limit the computational burden of the MPC scheme at the wind farm level, the structural tower dynamics (2) are also ignored (i.e., $\ddot{x}_{T}=\dot{x}_{T}=0$ ) because of the long time-scale of the wake interaction and the slow flow propagation. This simplification is reasonable since the load coordination law aims to react against slow wake-induced loadings rather than trying to damp the structural tower resonances.

Applying the forward Euler method to discretize (1) and (8), with a sample period $\Delta t$, yields the wind farm system matrix $\mathbf{A}_{k}$ and the control distribution 
matrices $\mathbf{B}_{k}$ and $\mathbf{D}_{k}$ with the following for their diagonal elements:

$$
\begin{aligned}
A_{i, k} & =1-\frac{\Delta t}{\tau_{i, k}}, \\
B_{i, k} & =2 \rho A_{d} U_{d_{i}, k}^{3} \frac{\Delta t}{\tau_{i, k}}, \\
D_{i, k} & =2 \rho A_{d} U_{d_{i, k}}^{2} h_{H},
\end{aligned}
$$

which vary with respect to the disc-averaged wind velocity $U_{d_{i}, k}$ at each time instant $k$, described as a function of the approximated effective wind speed (6) and the virtual axial induction factor (10) as:

$$
U_{d_{i}, k}=\frac{\hat{U}_{0_{i}, k}}{1+\beta_{i, k}} .
$$

The presented state-space model (11)-(12) was developed to be used as the prediction model of the MPC scheme. Two important features of the model should be highlighted due to its being a function of the disc-averaged wind velocity $U_{d_{i}, k}$ at the $i^{\text {th }}$ individual wind turbine. Firstly, the prediction model is updated at each sample time using direct measurements of the locally disc-averaged wind velocity $U_{d_{i}, k}$ from the LES representation of the wind farm simulation model, presented in section II. Therefore, the approximated effective wind speed $\hat{U}_{0_{i}, k}$ at different turbines reflects dominant dynamics of wind farm flows and wakes at each sample time. Secondly, the constructed prediction model (11)-(12) entails a parameter varying nature over the prediction horizon $N_{p}$ in the MPC architecture because of the dependency of $U_{d_{i}, k}$ on $\beta_{i, k}$ according to (16).

In this study, we consider only the load measurements of the tower base fore-aft bending moment, (i.e., $L_{i}=M_{T_{i}}$ ) as an indicator for intensified structural loading caused by wakes, and the other resonant responses are ignored. The wind turbine control can locally contribute to an increase in the structural damping on small time scales [44], [47], but this was not considered in this paper. Other load quantities (e.g., variations in the flap-wise blade loading, short-term damage equivalent loads (DELs) of the blade, main shaft response, and actuator wear and tear) should also be considered to be more representative for a real plant. The proposed load control approach could be applied in principle for more of such sophisticated descriptors if their measurements (or estimates) are available online.

\section{B. Optimal control problem}

We formulated the optimal control problem to simultaneously minimize the wind farm power reference tracking error and the deviations in the structural load of wind turbines from their mean values over a finite time prediction horizon $N_{p}$. Similar to [17], we exploited multiple solutions of the APC problem to achieve an equalized structural load distribution among the individual wind turbines, while the total power production of the turbines follows a wind farm power reference. Other load performance indices, e.g., minimization of the maximum loads, are also possible. Our control objective is to find an optimal feedback control law $\boldsymbol{\beta}_{k}^{\star}$ so that the system remains feasible and minimizes the average cost of interest (17) over the prediction horizon $N_{p}$. The proposed optimization MPC scheme solves the following constrained optimal control problem at each time instant $k$ :

$$
\begin{array}{ll}
\min _{\boldsymbol{\beta}_{k}} & \sum_{k=1}^{N_{p}} \mathscr{J}_{k}\left(\boldsymbol{P}_{k}, \boldsymbol{L}_{k}, \boldsymbol{\beta}_{k}\right), \\
\text { s.t. } & \boldsymbol{P}_{k+1}=\mathbf{A}_{k} \boldsymbol{P}_{k}+\mathbf{B}_{k} \boldsymbol{\beta}_{k}, \\
& \boldsymbol{L}_{k}=\mathbf{D}_{k} \boldsymbol{\beta}_{k}, \\
& \boldsymbol{\beta}_{N_{p}}=\boldsymbol{\beta}_{s s}^{\star}, \\
& 0 \leq \beta_{i, k} \leq \frac{1}{2}, \quad \equiv 0 \leq a_{i, k} \leq \frac{1}{3},
\end{array}
$$

with the following multi-objective performance index:

$$
\begin{aligned}
& \mathscr{J}_{k}\left(\boldsymbol{P}_{k}, \boldsymbol{L}_{k}, \boldsymbol{\beta}_{k}\right)= \\
& \frac{1}{2} \mu e_{\mathrm{P}, k}^{2}+\frac{1}{2}(1-\mu) \boldsymbol{e}_{\mathrm{L}, k}^{T} \boldsymbol{e}_{\mathrm{L}, k}+\frac{1}{2} \Delta \boldsymbol{\beta}_{k}^{T} \mathbf{R} \Delta \boldsymbol{\beta}_{k},
\end{aligned}
$$

which firstly addresses the square of the total wind farm power reference tracking error $e_{\mathrm{P}, k} \in \mathbb{R}$ at time instant $k$ as:

$$
e_{\mathrm{P}, k}=P_{k}^{\mathrm{ref}}-\sum_{i=1}^{N_{t}} P_{i, k} \text {. }
$$

Secondly, the index addresses the quadratic criterion of the instant structural load deviations based on the definition of a load-based tracking error $\boldsymbol{e}_{\mathrm{L}, k} \in \mathbb{R}^{N_{t}}$ at time instant $k$ as [17]:

$$
\boldsymbol{e}_{\mathrm{L}, k}=\left(\frac{1}{N_{t}} \sum_{i=1}^{N_{t}} L_{i, k}\right) \mathbf{1}_{N_{t} \times 1}-\boldsymbol{L}_{k},
$$

where $\mathbf{1}_{N_{t} \times 1}$ is defined as $[11 \ldots 1]^{T} \in \mathbb{R}^{N_{t}}$. The controller aims for the wind turbines to have equal tower base moments independent from different wake disturbances. As a result, load responses to the intensified wind velocity fluctuations of wakes are smoothed and the fatigue loads are reduced. The design parameter $\mu \in \mathbb{R}, 0 \leq \mu \leq 1$ is introduced to specify the relative importance of the wind farm power tracking accuracy and the amount of structural load equalization. Finally, the last term of (22) provides an assessment of the deviation of the control input with $\Delta \boldsymbol{\beta}_{k}=\boldsymbol{\beta}_{k}-\boldsymbol{\beta}_{k-1} \in \mathbb{R}^{N_{t}}$, where $\boldsymbol{\beta}_{0}$ for $k=1$ is set to the last applied control signal obtained at the previous optimization window. The weighting matrix $\mathbf{R} \in \mathbb{R}^{N_{t} \times N_{t}}$ is another design parameter used to penalize the control efforts for smooth and realizable induction control of wind turbines.

The equality constraints (18)-(19) represent the simplified wind farm model over the prediction horizon $N_{p}$. The final constraint (20) is introduced as the steady-state optimal solution $\boldsymbol{\beta}_{s s}^{\star}$ to enforce the trajectories $e_{\mathrm{P}, k}$ and $\boldsymbol{e}_{\mathrm{L}, k}$ to steer toward the origin and to avoid instability issues. Finally, the inequality constraint (21) represents practical constraints on the wind turbine control inputs; $\beta_{i, k}=0$ (i.e., $a_{i, k}=0$ ) represents the situation with zero-generated power, and $\beta_{i, k}=\frac{1}{2}$ (i.e., $a_{i, k}=\frac{1}{3}$ ) corresponds to the Betz limit [45] of the maximum extractable power of an isolated wind turbine. 


\section{Solving the optimization problem}

The parameter-varying nature of the prediction model (11)-(12) along with the existing non-linearities with respect to the scheduling variable $U_{d_{i}, k}$ cause the analytical expansion of the future state and control trajectories to be challenging and nontrivial for solving the formulated optimization problem. We estimate the effective wind velocity $U_{0}$ of the $i^{\text {th }}$ wind turbine at each sample time using three local measurements: (i) the disc-averaged wind velocity $U_{d_{i}, k}$ from PALM, (ii) the tower-top fore-aft velocity $\dot{x}_{T_{i}, k}$, and (iii) the applied axial induction factor $a_{i, k}$. The axial disc-averaged wind velocity $U_{d_{i}, k}$ is the main scheduling parameter of the system matrices; therefore, this velocity should vary with the corresponding control input trajectory $\beta_{i, k \mid k+N_{p}}$ so that the induction effect (6) is maintained over the specified prediction horizon $N_{p}$. We employ the adjoint method as an efficient tool for the online computation of the gradient to iteratively predict the optimal control trajectories $\beta_{i, k}^{\star}$. Similar to [20], the gradient calculation benefits from the computational efficiency of the adjoint approach, and yields the same computational cost as the low-order prediction model.

Based on [20], we construct an adjoint system having the same order as the prediction model (see Appendix A for details). Together with (11) and (12), this system forms a dual discretized system in the $2 N_{t}$ components of $\boldsymbol{Y}_{k}=\left[\begin{array}{ll}\boldsymbol{P}_{k}^{T} & \boldsymbol{L}_{k}^{T}\end{array}\right]^{T}$ and the $2 N_{t}$ components of the adjoint variable $\boldsymbol{\lambda}_{\boldsymbol{k}}=\left[\begin{array}{ll}\boldsymbol{\lambda}_{P, k}^{T} & \boldsymbol{\lambda}_{L, k}^{T}\end{array}\right]^{T}$. The backward time-propagation of the adjoint variable and the adjoint-based MPC gradient are derived over the prediction horizon $N_{p}$ as follows (see Appendix A for details):

$$
\begin{aligned}
\boldsymbol{\lambda}_{P, k} & =-\mathscr{J}_{\boldsymbol{P}_{k}}^{T}+\mathbf{A}_{k}^{T} \boldsymbol{\lambda}_{P, k+1}, \\
\boldsymbol{\lambda}_{L, k} & =-\mathscr{J}_{\mathbf{L}_{k}}^{T}, \\
\nabla_{\boldsymbol{\beta}_{k-1}} \mathscr{J} & =\Delta \boldsymbol{\beta}_{k-1}^{T} \mathbf{R}+\boldsymbol{\lambda}_{P, k}^{T}\left(\boldsymbol{C}_{k}\right)_{\boldsymbol{\beta}_{k-1}}+\boldsymbol{\lambda}_{L, k}^{T}\left(\boldsymbol{E}_{k}\right)_{\boldsymbol{\beta}_{k-1}}
\end{aligned}
$$

where $\mathscr{J}_{\boldsymbol{P}_{k}}$ and $\mathscr{J}_{\boldsymbol{L}_{k}}$ represent the partial derivatives of the performance index (22) with respect to the wind turbines' power production $\boldsymbol{P}_{k}$ and the structural load $\boldsymbol{L}_{k}$. The terms $\left(\boldsymbol{C}_{k}\right)_{\boldsymbol{\beta}_{k-1}} \in \mathbb{R}^{N_{t} \times N_{t}}$ and $\left(\boldsymbol{E}_{k}\right)_{\boldsymbol{\beta}_{k-1}} \in \mathbb{R}^{N_{t} \times N_{t}}$ represent the partial derivatives of the prediction model (11)-(12) with respect to the control input, which are expanded in Appendix A over the prediction horizon. Indeed, the adjoint variable associates the variations of the performance index and the prediction model over the prediction horizon $N_{p}$. This means that we are faced with a two-point boundary value problem. While the control variable is initialized with the optimal solution of the previous optimization window $\left(\boldsymbol{\beta}_{k-1}^{\star}\right)$, the adjoint variable is initialized at the end of the prediction interval with the solution of the steady-state adjoint equation $\left(\boldsymbol{\lambda}_{s s}^{\star}\right)$ for addressing the final constraint (20).

We solve the optimal control problem iteratively at each prediction window. Given the instant control variable $\boldsymbol{\beta}_{k}^{(n)} \in$ $\mathbb{R}^{N_{t}}$ at the $n^{\text {th }}$ optimization iteration, we determine a new estimation at the time instant $k$ using the adjoint-based gradient of (27) as follows:

$$
\boldsymbol{\beta}_{k}^{(n+1)}=\boldsymbol{\beta}_{k}^{(n)}+\eta^{(m)}\left(\nabla_{\boldsymbol{\beta}_{k}}^{(n)} \mathscr{J}\right)^{T}
$$

where $\eta^{(m)}$ is the step size along a given search direction, which ensures that an improvement is achieved in the total power production at each optimizing iteration. We employ a backtracking line search method to find the proper step size that was initialized with its maximum value $\eta^{(0)}=1$. Until the improvement in the performance index (17) is achieved, the step size at the $m^{\text {th }}$ line search iteration is set as:

$$
\eta^{(m)}=\frac{1}{2} \eta^{(m-1)} .
$$

Moreover, an active set method is employed for applying the inequality constraint (21) and maintaining the applied control inputs within the practical constraints of the wind turbines. More details on the adjoint-based MPC architecture are available in [20].

\section{Receding horizon control law}

We obtain the optimal solution when the gradient falls below a user-defined threshold. Based on receding horizon control, we apply the first part of the optimal solution to the wind power plant over the receding horizon $N_{u} \leq N_{p}$. Note that the optimal solution $\boldsymbol{\beta}_{k}^{\star}$ is transferred back into the axial induction factors using (10) and applied to individual wind turbines for simultaneous power and load regulation while their total power follows the reference signal demanded by the TSO (see Fig. 2). In practice, the induction factor should be translated to the turbine power reference when passed to the turbine controller [15], [16], which is out of the scope of this paper. Repeating the whole procedure at the next sample time with new measurements provides feedback into the optimal control problem, which enables the AMPC to react to varying atmospheric and operating conditions. The length of $N_{u}$ determines how fast the wind farm responds to the disturbances [48]. Increasing $N_{u}$ degrades the closed-loop performance and makes the control system less responsive to fast dynamics such as wake meandering, wind gusts, and turbulent flow [20].

\section{RESULTS AND DISCUSSION}

This section focuses on the simulation scenario, in which the wake interactions are problematic for good wind farm power-tracking performance, similar to [8], [9], [17]. In non-wake conditions, wind turbines do not aerodynamically influence each other, which simplifies the control problem; we do not address this condition. The following three APC approaches are evaluated in this study:

1) A PI-based APC (hereinafter PI-Ref. APC) [9],

2) A PI-based APC with a CLD law (hereinafter PIAPC/CLD) [17],

3) The proposed AMPC-based APC with CLD (hereinafter AMPC-APC/CLD) for optimal structural load equalization. 


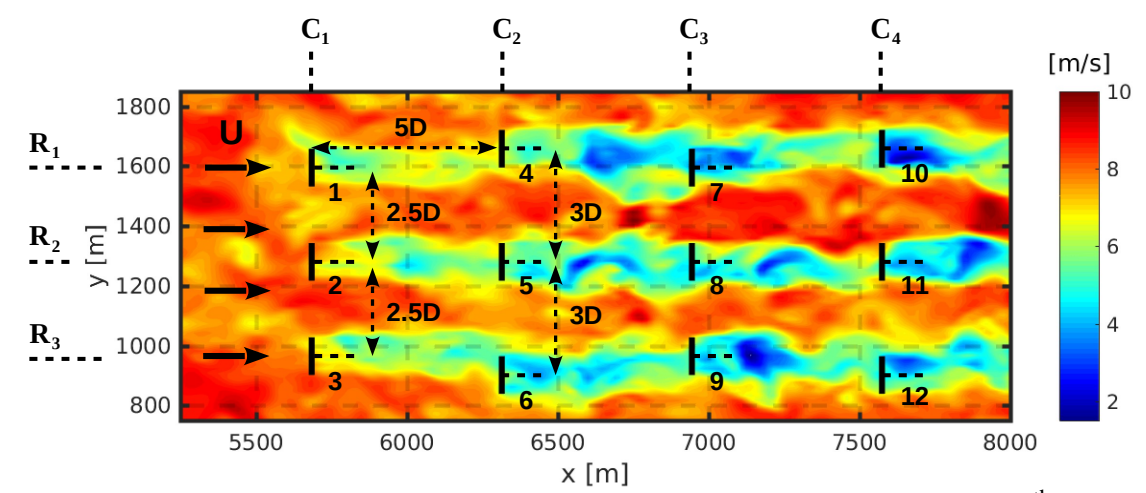

Fig. 3. Layout of the waked $3 \times 4$ wind farm model simulated using PALM. $\mathrm{R}_{i}$ and $\mathrm{C}_{j}$ stand for the $i^{\text {th }}$ row and the $j^{\text {th }}$ column of the simulated wind turbines.

TABLE II

KEY PARAMETERS OF THE PALM [41] SIMULATION SET-UP.

\begin{tabular}{lc}
\hline Simulation parameter & Value \\
\hline Domain size $L_{x} \times L_{y} \times L_{z}$ & $15.3 \times 3.8 \times 1.3 \mathrm{~km}^{3}$ \\
Grid mesh size $N_{x} \times N_{y} \times N_{z}$ & $1024 \times 256 \times 128$ \\
Cell mesh resolution $\Delta_{x} \times \Delta_{y} \times \Delta_{z}$ & $15 \times 15 \times 10 \mathrm{~m}^{3}$ \\
Number of wind turbines $N_{t}$ & 12 \\
Wind turbine model & Actuator disc model (ADM) \\
Wind turbine control degree of freedom & Induction factor \\
Number of grid cells per turbine & 68 \\
Atmospheric stability condition & Neutral boundary layer $(\mathrm{NBL})$ \\
Ambient longitudinal wind speed $U_{\infty}$ at hub height & $8 \mathrm{~m} / \mathrm{s}$ \\
Geostrophic wind velocity & $u_{g}=9 \mathrm{~m} / \mathrm{s}$ and $v_{g}=-2 \mathrm{~m} / \mathrm{s}$ \\
Monin-Obukhov length scale & $3.8 \mathrm{~km}$ \\
Longitudinal turbulence intensity of ambient wind & $I_{u} \approx 5 \%$ \\
Longitudinal turbulence intensity of wakes & $I_{u} \approx 15 \%$ \\
30-min averaged wind farm power $\left(P_{\text {farm }}^{\text {base }}\right)$ & $12.3 \mathrm{MW}$ \\
Simulation sample time & $1 \mathrm{~s}$ \\
\hline
\end{tabular}

\section{A. Case study}

We consider a reference layout of a $3 \times 4$ wind farm with different wake overlaps with downstream wind turbines used in [17] (see Fig. 3). The parameters of the wind turbine models are taken from the freely available model of the NREL $5 \mathrm{MW}$ reference wind turbine with a rotor diameter of $D=126 \mathrm{~m}$ and a hub height $h_{H}=90 \mathrm{~m}$ [49]. The wind turbines are spaced five rotor diameters $(5 D)$ in the stream-wise direction. In the first and third rows (i.e., turbines $\{1,4,7,10\}$ and $\{3,6,9,12\})$, the rotor centers of the downstream turbines are intentionally offset half a rotor diameter from the centers of their upwind turbines, i.e., partial wake conditions. The wind turbines in the middle row (i.e., turbines $\{2,5,8,11\}$ ) are spaced without lateral offset to create full wake interactions. Fig. 3 shows the instantaneous field of the $u$-component of the wind at the hub height of the wind turbines, and we clarify the conventions that we use for the rows $\left(R_{1}\right.$ to $\left.R_{3}\right)$ and columns $\left(C_{1}\right.$ to $\left.C_{4}\right)$ of the wind farm.

We simulated a neutral boundary layer (NBL) with a capping inversion [38] and a mean wind speed of $8 \mathrm{~m} / \mathrm{s}$ at the hub height along the $\mathrm{x}$-axis of the simulation domain. Note that the geostrophic wind velocities $u_{g}=9 \mathrm{~m} / \mathrm{s}$ and $v_{g}=-2 \mathrm{~m} / \mathrm{s}$ were defined empirically and iteratively to result in the ambient wind velocity $U_{\infty}=8 \mathrm{~m} / \mathrm{s}$ at the hub height. A precursor simulation of the atmospheric boundary layer was first conducted without any turbines to allow for the generation of a fully developed undisturbed turbulent flow field. Then, the flow field was used for the initialization of the main wind farm simulation runs in which a turbulence recycling method was used [41]. The longitudinal turbulence intensities at the hub height were approximately $5 \%$ at the turbine location and $15 \%$ at the $5 D$ distance downstream in the wake center. Table II summarizes the parameters of the PALM simulation set-up.

We conducted a wind farm power reference tracking scenario to evaluate the APC performance of the simulated wind farm using PALM. The time-varying TSO power reference used was the same as that in [17]:

$$
P_{k}^{\mathrm{ref}}=P_{\mathrm{farm}}^{\mathrm{base}}\left(b+c n_{k}^{\mathrm{AGC}}\right),
$$

where $P_{\text {farm }}^{\text {base }}=12.3 \mathrm{MW}$ was obtained based on the timeaveraged available power of the wind farm with the local greedy control setting of $a_{i}=\frac{1}{3}$ for each turbine. To generate the normalized perturbation of $n_{k}^{\mathrm{AGC}}$, we employed the normalized RegD type of automatic generation control (AGC) signal, that is, the most rapidly actuating test signal used for APC qualification by a regional transmission organization in the eastern United States [50]. The signal was parameterized here with $b=0.8$ and $c=0.1$ indicating that the mean 
power reference is $80 \%$ of the mean available power and the reference signal may be perturbed by up to $10 \%$ of the mean available power (see Fig. 4).

\section{B. Active power control approaches}

We considered two recently developed closed-loop APC approaches here as the baselines to show the effectiveness of the proposed MPC approach. First, following [9], a PI-based APC (PI-Ref. APC) was designed to improve the wind farm power tracking performance by resolving the undesirable local effects of the turbulent wind farm flows and wakes. Second, a PI-based distribution law termed APC/CLD [17] was employed to equalize the wind turbines structural loading during wind farm power reference tracking.

In both approaches, the PI-based control signal $\Delta P_{k}^{\text {ref }} \in \mathbb{R}$ actively adjusts the wind turbine power demands $\boldsymbol{P}_{k}^{\mathrm{dem}} \in \mathbb{R}^{N_{t}}$ to cooperatively compensate for the accumulated local power tracking errors at each time instant $k$ given as [17]

$$
\boldsymbol{P}_{k}^{\mathrm{dem}}=\boldsymbol{\alpha}_{k}\left(P_{k}^{\mathrm{ref}}+\Delta P_{k}^{\mathrm{ref}}\right),
$$

where the distributing power set-points $\boldsymbol{\alpha}_{k} \in \mathbb{R}^{N_{t}}$ can be chosen freely within the constraint $\sum_{i=1}^{N_{t}} \alpha_{i, k}=1$ where $0 \leq \alpha_{i, k} \leq 1$. Contrary to PI-Ref. APC with pre-selected power set-points $\alpha_{i, k}=\frac{1}{12}$, the PI-APC/CLD exploits the non-uniqueness of the APC solution to alleviate the wakeinduced structural fatigue loading. A PI-based power distribution law was designed to adjust the power set-points for leveling the structural loadings of the individual wind turbines. More details on APC with CLD and its design parameterization are available in [17].

The design parameters of the formulated AMPC in Section III were chosen as the prediction horizon $N_{p}=60$ with the sample time of $1 \mathrm{~s}$. The first sample of the optimal solution was implemented according to the conventional receding horizon principle, that is, $N_{u}=1$. The parameter $\mu$ defines the importance of power tracking relative to load equalization. When there is enough available power, the value of $\mu$ does not influence the performance significantly. For instance, in the current study, with the mean wind farm power reference being $80 \%$ of the mean available power, $\mu=0.8$ was chosen, but no differences in behavior were observed for $\mu=0.7$ or $\mu=0.9$. When the power reference is closer to the available power, e.g., above $90 \%$, more attention should be given to tuning the $\mu$ parameter [17]. The weighting matrix on the control effort was chosen as $\mathbf{R}=0.5 \mathbf{I}_{12}$ to avoid large changes in the axial induction factors, where $\mathbf{I}_{12}$ is the identity matrix with dimensions equal to the number of wind turbines. We assumed that the sampled AGC signal remained constant over the prediction window.

\section{Evaluation of control approaches}

We conducted a comparative study for investigating an optimal structural load distribution among the individual wind turbines while their total power production followed the power reference required by the TSO.

Fig. 4 plots the AGC response and indicates the root mean square (RMS) of the wind farm power tracking error from $300 \mathrm{~s}$ to $2100 \mathrm{~s}$ for the examined APC approaches. Our analyses were focused on the AGC signal after $300 \mathrm{~s}$ to allow time for inflow propagation and wake development. APC and structural load coordination were activated at $200 \mathrm{~s}$ and $300 \mathrm{~s}$, respectively. All the examined APC approaches achieved a satisfactory wind farm power reference tracking performance. However, the tracking accuracy, given by the root mean square (RMS) of the tracking errors in the legend of Fig. 4, was further improved when the wind turbines' structural loads were levelized using both the PI-APC/CLD and AMPC-APC/CLD.

The effect can be explained from Figs. 5-8. Figures 5 and 6 reveal the impact of the formulated predictive optimal control for coordinating the structural loads of the individual wind turbines during wind farm power reference tracking (Fig. 4). Fig. 5 displays the RMS of the errors across all wind turbines between the measured tower base fore-aft bending moments and their mean value, which were calculated at each time instant. The standard deviations (STD) of the tower base fore-aft moment of all

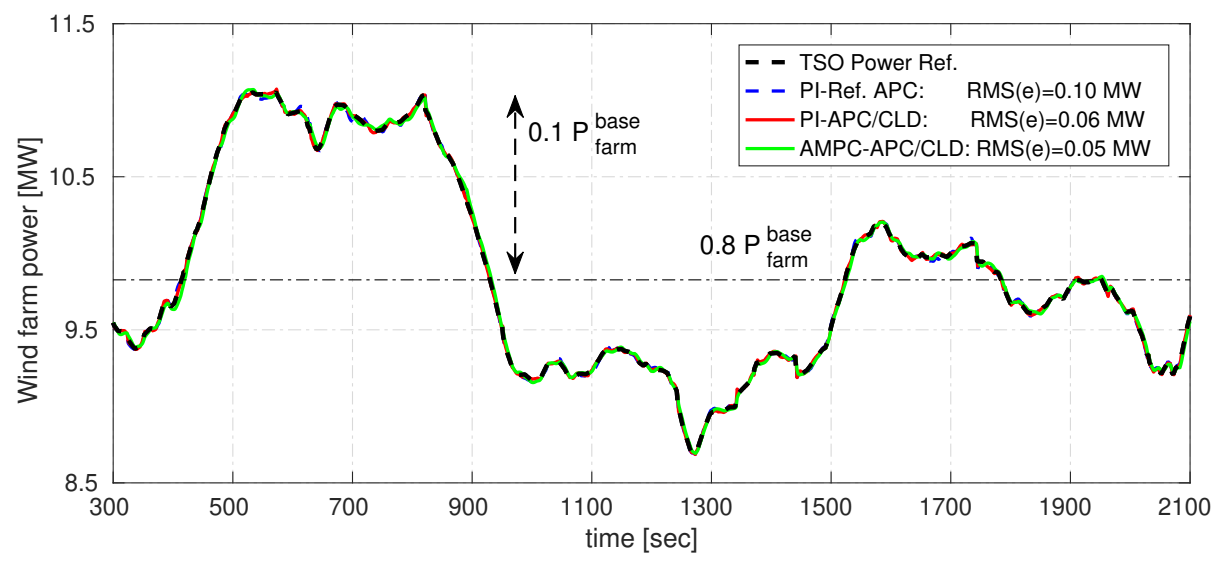

Fig. 4. Total power production of the wind farm with AMPC-based APC with CLD, as compared with the classical control approaches. The time-varying power reference (30) was parameterized with $P_{\text {farm }}^{\text {base }}=12.3 \mathrm{MW}, b=80 \%$, and $c=10 \%$. All the curves are nearly coincident with one another. 


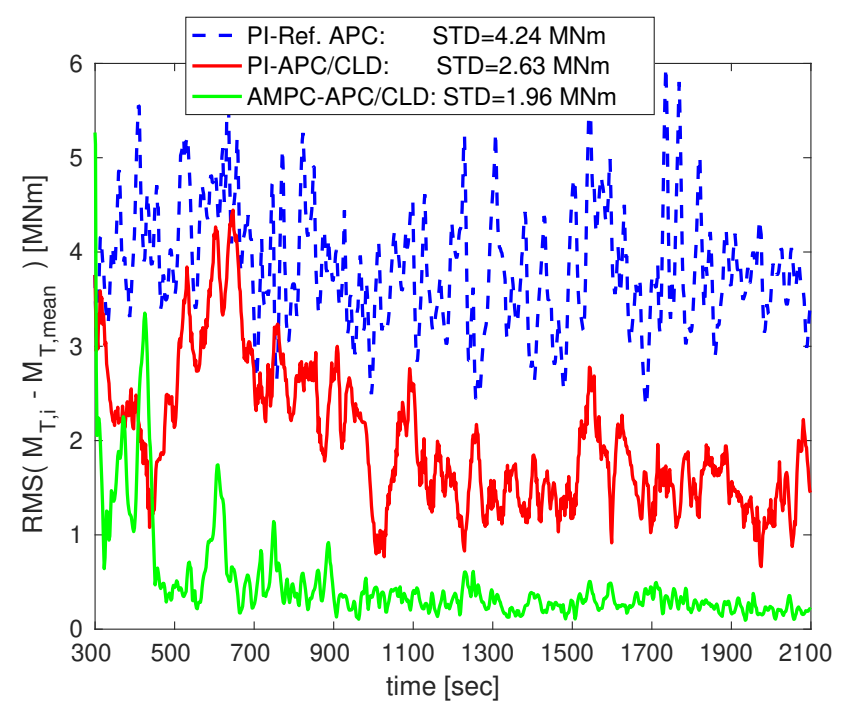

Fig. 5. RMS errors between the tower base fore-aft bending moment of the individual wind turbines and their mean values for the examined APC approaches.

wind turbines from $300 \mathrm{~s}$ to $2100 \mathrm{~s}$ are also noted in the legend for each approach. In [17], the authors showed that the RMS values of the load-based errors were significantly reduced by employing the CLD law over time (see the red curve in Fig. 5). However, the proposed AMPC-APC/CLD is capable of reducing the structural load deviations further by optimally coordinating the wind turbines' power production.

Fig. 6 compares the STD of the tower base fore-aft bending moment of the individual wind turbines from $300 \mathrm{~s}$ to 2100 s. The PI-APC/CLD (see red bars in Fig. 6) reduces the load deviations of the downwind turbines operating inside the wakes compared with the load deviations of PI-Ref. APC.

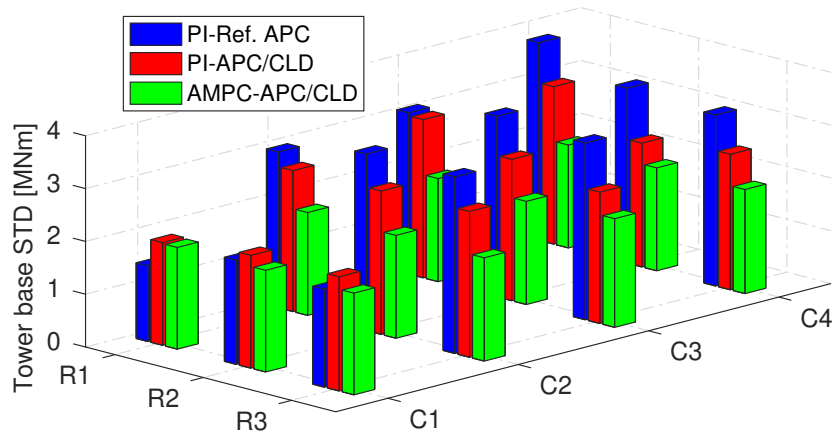

Fig. 6. STD of the tower base fore-aft bending moment of the individual wind turbines for the different APC approaches. R and $\mathrm{C}$ stand for the row and column of the wind turbines, respectively (see Fig. 3).

However, we can see how the AMPC scheme exploits the flexibility of the APC problem for almost complete equalization of the structural loading of the wind turbines, regardless of operations in waked or ambient conditions.
Figs. 7 and 8 depict the contribution of the individual wind turbines in the wind farm power tracking performance (shown in Fig. 4) for the three examined APC approaches over the time span up to $1300 \mathrm{~s}$. Fig. 7 presents the trajectories of the axial induction factors of the individual wind turbines. The PI-based Ref. APC mostly saturates the downwind turbines $\{8,10,11,12\}$ to the greedy operating point $a_{i}=\frac{1}{3}$ (see dashed blue curves in Fig. 7), which means that they do not contribute to rejecting the high wind velocity fluctuations of wakes as the sources of the fatigue loads. The PI-APC/CLD actively adjusts the wind turbine power demands (31) to transfer wake-induced loadings from the downwind turbines to the turbines exposed to low dynamic loadings, particularly the upwind turbines. Limiting the power demand increases their controllability. As shown, the axial induction factors are saturated less often and hence have more freedom (see red curves in Fig. 7) to reject the dynamic loading. However, the two classically designed control loops in the PI-APC/CLD might inefficiently compete with each other, particularly when the wind farm power demand is high [17]. With the AMPC-APC/CLD (see green curves in Fig. 7), the wind turbine power productions are optimally adjusted according to their locally available power. Therefore, they cooperatively avoid wind turbine control saturation while an APC solution exists.

Fig. 8 compares the corresponding power contributions of the individual wind turbines to the wind farm power reference tracking, shown in Fig. 4. The power production of the waked wind turbines $\{8,10,11,12\}$ with the PI-based Ref. APC mainly reflects the fluctuations of the turbulent flow inside the wakes mostly because of operating with the local greedy control of $a_{i}=\frac{1}{3}$ (see dashed blue curves in Fig. 7). The power fluctuations are reduced by actively adjusting the power set-points of the wind turbines with the PI-APC/CLD (see red curves). The green curves show how the power productions are regulated with AMPC-APC/CLD to yield an equalized structural load coordination.

Finally, the effectiveness of the proposed MPC scheme was evaluated using fatigue load analysis. Structural fatigue loading is among the key factors in the design of wind turbines [51]. Fatigue load analysis was achieved by comparing the load spectra obtained from rainflow-counting of the time series of the stresses with a characteristic curve of the design resistance of a component called the $\mathrm{S}-\mathrm{N}$ or Wöhler curve. This study only provides a qualitative assessment of the corresponding damage equivalent load (DEL) of the tower base fore-aft bending moment as a descriptor for the structural fatigue loading.

Fig. 9 depicts the corresponding DELs of the fore-aft tower base bending moment of all twelve wind turbines for the examined APC approaches. These loads were computed using the 30-min time series (from $300 \mathrm{~s}$ to $2100 \mathrm{~s}$ ). The inverse slope of the S-N curve was considered to be $m=4$, which is commonly used for steel components similar to a tower. The reference number of the cycles for computing the DELs was taken as $2 \times 10^{6}$. The PI-based CLD law 

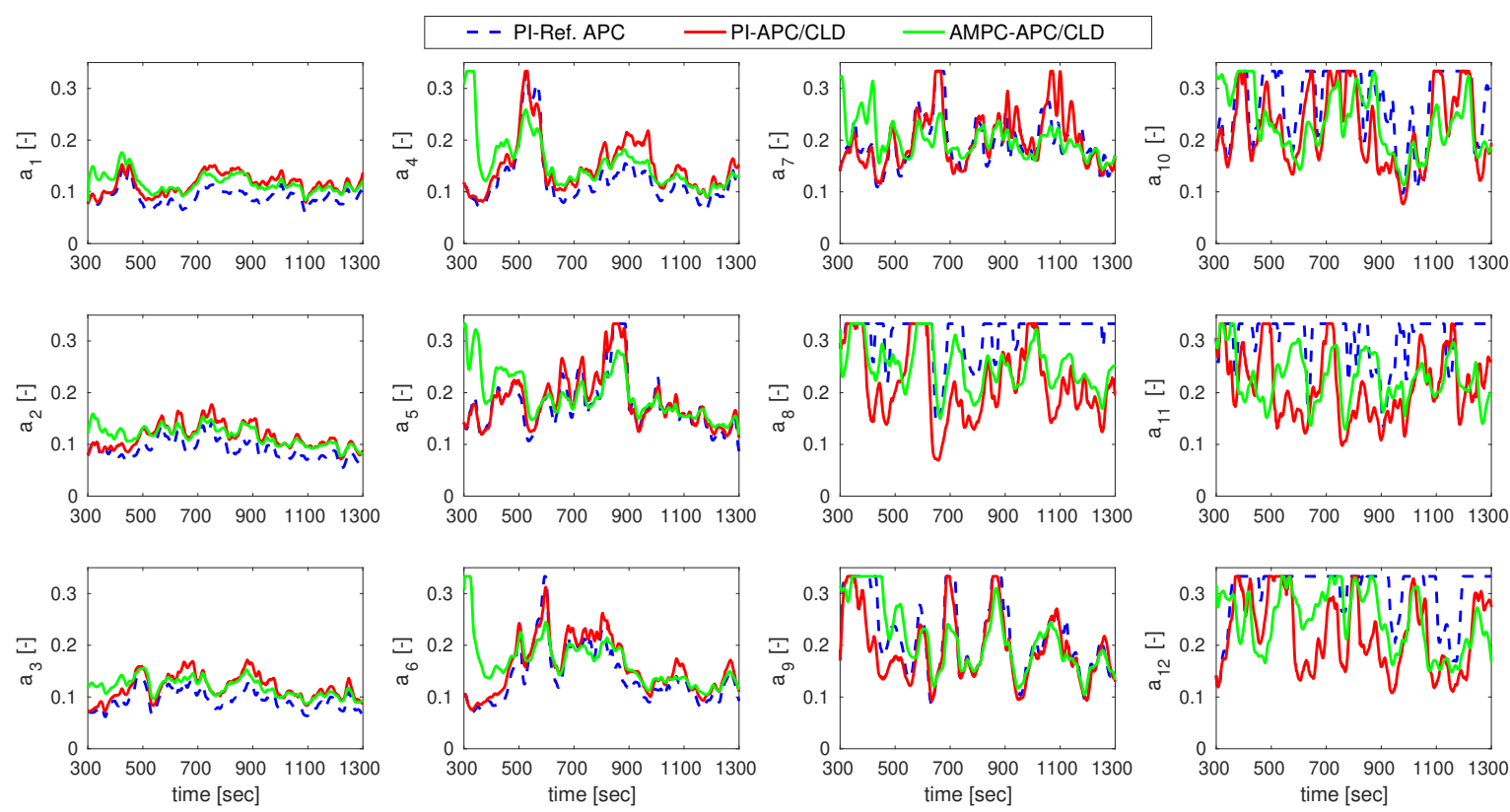

Fig. 7. The axial induction factor trajectories of the individual wind turbines with different closed-loop APC strategies for tracking the power reference with $P_{\text {farm }}^{\text {base }}=12.3 \mathrm{MW}, b=80 \%$, and $c=10 \%$. Both the PI-based and the AMPC-based APC with CLD actively equalize the individual wind turbine loadings during wind farm power reference tracking.
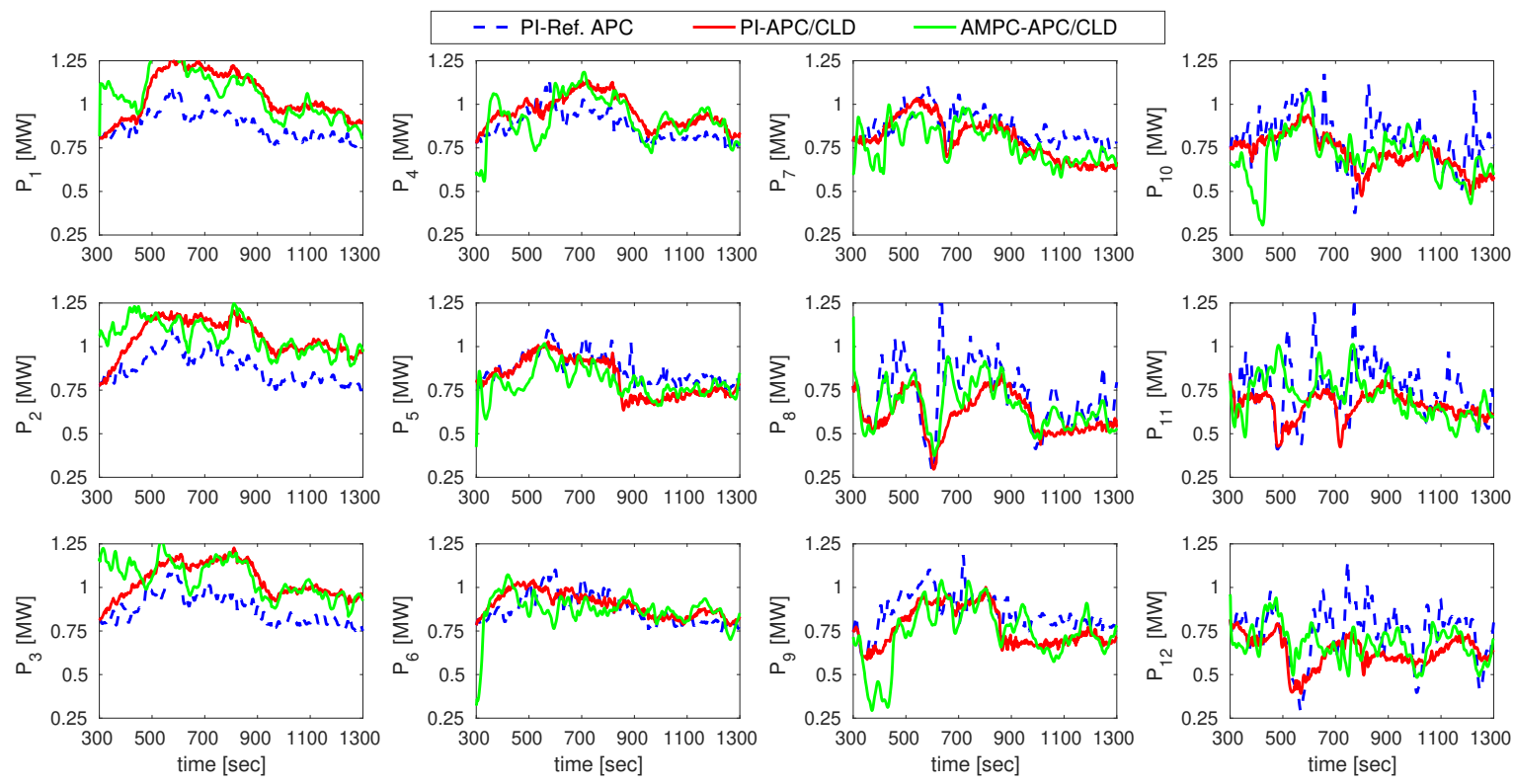

Fig. 8. The power productions of the individual wind turbines with different closed-loop APC strategies for tracking the power reference with $P_{\mathrm{farm}}^{\text {base }}=12.3 \mathrm{MW}, b=80 \%$, and $c=10 \%$. Both the PI-based and the AMPC-based APC with CLD actively equalize the individual wind turbine loadings during wind farm power reference tracking.

significantly mitigated the fatigue loading of the waked wind turbines in return for a slight increase in the upwind turbines $\{1,2,3\}$. The AMPC-APC/CLD equalizes the structural loading of the wind turbines by better distribution of the TSO power reference among the individual turbines. The mean DEL is also indicated in the legend to evaluate the wind farm fatigue loading as a whole. However, the local quantities are important for assessing particular turbine situations with high DELs that are not balanced by other periods with low DELs because of the strong non-linearity of the fatigue damage on the stress amplitude.

\section{Computational complexity analysis}

To consider the proposed MPC scheme for real-time implementation in wind tunnels and open field experiments, the computational effort of the controller should be taken into account. The computational complexity of the AMPC architecture is determined by the model order and the number of decision variables over the prediction horizon [20]. 


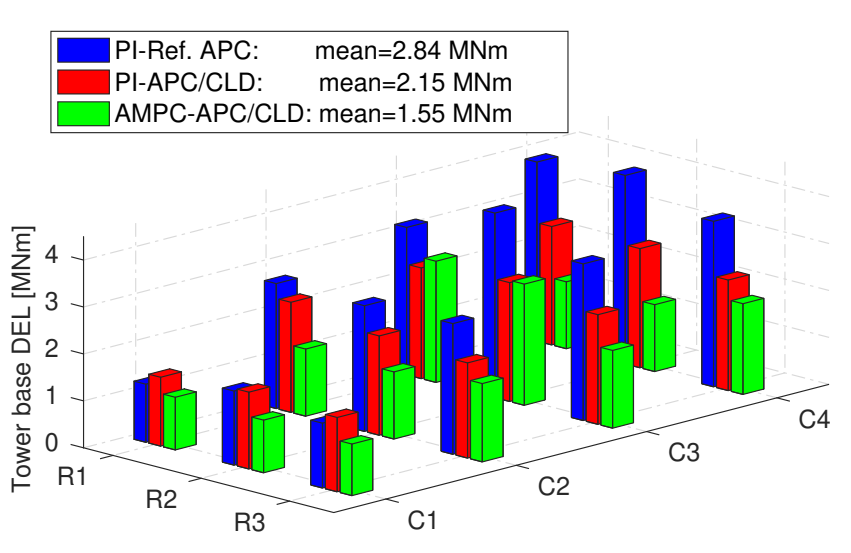

Fig. 9. Corresponding DELs of the tower base fore-aft bending moment of the individual wind turbines for the different APC approaches. The time-varying wind farm power reference (30) was parameterized with $P_{\text {farm }}^{\text {base }}=12.3 \mathrm{MW}, b=80 \%$, and $c=10 \%$. R and $\mathrm{C}$ are the row and column of the wind turbines (see Fig. 3).

We developed an AMPC-based APC in [22] which employed a medium-fidelity 2D dynamic wind farm model [29] to predict wake interactions at hub-height of wind turbines in advance. Although one can expect an optimal control performance using this approach, the required computational effort would be intractable for real-time control, especially for large wind farms. As discussed in Section III-A, the prediction model used in this paper neglects the wake propagation model, which significantly improves the computational efficiency of the AMPC scheme.

We performed a comparative study to evaluate the computational complexity of the presented AMPC-APC/CLD, which can be implemented for real-time control. The presented AMPC design in [22], with the wind farm flow prediction model of [29], is considered as a reference to highlight the importance of the updated design. A direct comparison of the performance on power tracking and load equalization between the two approaches is beyond the scope of this paper since [22] considered only laminar wind inflow while a turbulent wind field is taken into account in the present study. Nonetheless, from a qualitative point of view, both approaches are expected to deliver similar tracking and load reduction prospects. The striking difference of high relevance for a future application is given by the distinct computational complexity of both methods.

Table III shows the large improvement with respect to the elapsed computing time and summarizes the governing characteristics and parameterizations of the examined APC approaches with their prediction model components and orders. The elapsed time for the computation of a single receding horizon control is indicated for assessing the computational efficiency of the current MPC scheme for the APC with the equalized structural load coordination. The number of iterations of the AMPC is variable; therefore, it is limited to two iterations per control action for a better comparison of the controllers' performances. In the current case study with power level of $80 \%$ of the available power, this limit was not activated due to the large control solution domain. When the power reference is closer to the available power, more iterations and longer computing time are expected due to more frequent activations of constraints. For this study, the simulations were run on the EDDY high-performance computing cluster ${ }^{1}$ with a single core. Note that the elapsed computing time of the recently designed PI-APC/CLD [17] was $0.3 \mathrm{~ms}$. However, this does not guarantee the optimal closed-loop performance, and it is insufficient for addressing the multi-objective control problem and effectively handling the saturation constraints, as shown in Section IV-C.

The impact of simplifying the prediction model on the computational complexity of the AMPC is obvious enough. One important achievement is that the wake prediction model is not required for AMPC-APC/CLD because structural load equalization is achieved as shown in Section IV-C even though the aerodynamic coupling among the wind turbines is neglected during controller optimization. A possible explanation for this is that the unmodelled wake interactions act as an additional source of disturbance. As already shown in [17], the closed-loop APC can effectively compensate disturbances caused by the turbulent flows and wakes, which also leads to the high quality power tracking and load equalization in this study. The relative benefits of the proposed AMPC scheme as compared with the PI-APC/CLD for the optimal coordination of the structural loads of individual wind turbines have already been discussed in Section IV-C. Our future research will focus on the implementation of the AMPC-APC/CLD and the associated practical considerations for wind tunnel and open field experiments. A simple structure of the prediction model could allow the development of an explicit MPC to avoid the online computation of the gradient and to further improve the computational efficiency of the AMPC architecture; however, this is outside the scope of this study.

TABLE III

COMPUTATIONAL COMPLEXITY OF THE AMPC-BASED APC WITH CLD FOR EVALUATING REAL-TIME IMPLEMENTATION.

\begin{tabular}{lll}
\hline & $\begin{array}{l}\text { AMPC-based } \\
\text { design [22] }\end{array}$ & $\begin{array}{l}\text { AMPC } \\
\text { (this study) }\end{array}$ \\
\hline $\begin{array}{l}\text { Wake prediction model } \\
\text { Wind turbine prediction model }\end{array}$ & WFSim [29] & N.A. \\
Prediction model order & 19824 & ADM \\
$\begin{array}{l}\text { Prediction horizon } N_{p} \\
\text { Receding horizon } N_{u}\end{array}$ & $60 \mathrm{~s}$ & 12 \\
(controller sample time) & $1 \mathrm{~s}$ & $60 \mathrm{~s}$ \\
Elapsed computing time & $122.2 \mathrm{~s}$ & $0.22 \mathrm{~s}$ \\
\hline
\end{tabular}

\section{CONCLUSION}

We proposed an AMPC of waked wind farms for simultaneously tracking a wind farm power reference and optimally coordinating the structural loadings of the individual wind turbines. There exist multiple solutions for the APC problem

\footnotetext{
${ }^{1}$ https://uol.de/fk5/wr/hochleistungsrechnen/hpc-facilities/eddy
} 
with respect to the distribution of wind turbine power demands; therefore, a solution with reduced structural loading is possible. In contrast to the PI-based design [17], systematic MPC optimization allows a multi-objective performance index to be defined to account for following a total power production reference and structural load coordination with efficient constraint handling. Contrary to the AMPC designs in [20] and [22], the computational complexity of the AMPC of this paper is significantly reduced by neglecting the spatial wake propagation model in the MPC design. Indeed, the optimal control problem was based on a model composed of decoupled wind turbine models, which aimed to predict the individual wind turbine power/load responses using direct local measurements from the turbulent wind farm flows and wakes, independent of their complexities. The MPC design was evolved to optimize input/output trajectories at each sample time for optimal distribution of the wind farm available power among the individual wind turbines. The MPC scheme benefits from the adjoint approach for the online computation of the gradient of the constrained optimization problem, whose system matrices vary over the prediction horizon with respect to the control input trajectories.

We examined the performance of the AMPC-based APC with CLD for structural load equalization by using a representation of the wind power plant by an LES model and compared the performance with the recently designed PIbased APC with CLD law [17]. The high-fidelity simulation results highlight that the AMPC scheme can simultaneously realize different control objectives and handle the practical constraints in a systematic way. Moreover, we demonstrated significant improvements in the wind farm behavior and wake-induced load alleviation could be achieved even with a simple numerical wind farm model, without modelling flow propagation or wakes in the wind farm. The current AMPC architecture features a computationally efficient MPC design with very simple sensory system requirements, which makes it possible to achieve real-time control of wind farms.

\section{APPENDIX A}

\section{DERIVATION OF THE ADJOINT-BASED MPC GRADIENT}

The constrained optimization problem (17)-(19) is rewritten here over the prediction horizon $N_{p}$ as

$$
\begin{array}{ll}
\min _{\tilde{\boldsymbol{\beta}}} & \mathscr{J}(\tilde{\boldsymbol{P}}, \tilde{\boldsymbol{L}}, \tilde{\boldsymbol{\beta}})=\sum_{k=1}^{N_{p}} \mathscr{J}_{k}\left(\boldsymbol{P}_{k}, \boldsymbol{L}_{k}, \boldsymbol{\beta}_{k}\right), \\
\text { s.t. } & \tilde{\boldsymbol{C}}(\tilde{\boldsymbol{P}}, \tilde{\boldsymbol{\beta}})=\mathbf{0}, \\
& \tilde{\boldsymbol{E}}(\tilde{\boldsymbol{L}}, \tilde{\boldsymbol{\beta}})=\mathbf{0} .
\end{array}
$$

The equality constraints (33)-(34) represent the wind turbines' power and structural load prediction models, which are subject to wind farm flows and wakes over the prediction horizon $N_{p}$ using the following expanded forms:

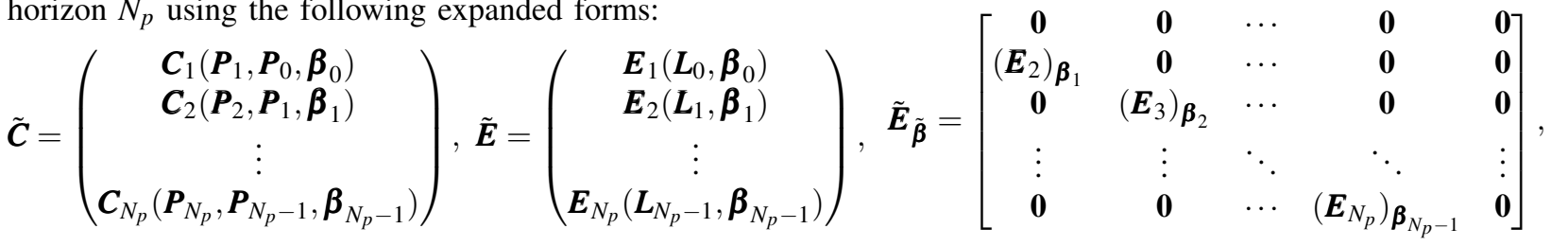

First, we define the Lagrangian to turn the constrained optimization problem (32)-(34) into an unconstrained one:

$$
\mathscr{L}(\tilde{\boldsymbol{P}}, \tilde{\boldsymbol{L}}, \tilde{\boldsymbol{\beta}}, \boldsymbol{\Lambda}) \equiv \mathscr{J}(\tilde{\boldsymbol{P}}, \tilde{\boldsymbol{L}}, \tilde{\boldsymbol{\beta}})+\boldsymbol{\Lambda}^{T}\left[\begin{array}{l}
\tilde{\boldsymbol{C}}(\tilde{\boldsymbol{P}}, \tilde{\boldsymbol{\beta}}) \\
\tilde{\boldsymbol{E}}(\tilde{\boldsymbol{L}}, \tilde{\boldsymbol{\beta}})
\end{array}\right],
$$

where $\boldsymbol{\Lambda}=\left[\boldsymbol{\Lambda}_{P}^{T} \boldsymbol{\Lambda}_{L}^{T}\right]^{T} \in \mathbb{R}^{2 N_{t} \times N_{p}}$ is the vector of Lagrange multipliers. The equality constraints (33)-(34) hold always for $k=1,2, \cdots, N_{p}$; therefore, we may choose $\boldsymbol{\Lambda}$ freely, and the gradient of the cost function can be expressed as

$$
\begin{aligned}
\nabla_{\tilde{\boldsymbol{\beta}}} \mathscr{J}=\nabla_{\tilde{\boldsymbol{\beta}}} \mathscr{L} & =\left(\mathscr{J}_{\tilde{\boldsymbol{P}}}+\boldsymbol{\Lambda}_{P}^{T} \tilde{\boldsymbol{C}}_{\tilde{\boldsymbol{P}}}\right) \tilde{\boldsymbol{P}}_{\tilde{\boldsymbol{\beta}}} \\
& +\left(\mathscr{J}_{\tilde{\boldsymbol{L}}}+\boldsymbol{\Lambda}_{L}^{T} \tilde{\boldsymbol{E}}_{\tilde{L}}\right) \tilde{\boldsymbol{L}}_{\tilde{\boldsymbol{\beta}}} \\
& +\mathscr{J}_{\tilde{\boldsymbol{\beta}}}+\boldsymbol{\Lambda}_{P}^{T} \tilde{\boldsymbol{C}}_{\tilde{\boldsymbol{\beta}}}+\boldsymbol{\Lambda}_{L}^{T} \tilde{\boldsymbol{E}}_{\tilde{\boldsymbol{\beta}}},
\end{aligned}
$$

where $(.)_{\tilde{\boldsymbol{P}}},(.)_{\tilde{L}}$, and $(.)_{\tilde{\boldsymbol{\beta}}}$ represent the partial derivatives with respect to $\tilde{\boldsymbol{P}}, \tilde{\boldsymbol{L}}$, and $\tilde{\boldsymbol{\beta}}$, respectively. The MPC gradient might be simplified to the following compact form:

$$
\nabla_{\tilde{\boldsymbol{\beta}}} \mathscr{J}=\mathscr{J}_{\tilde{\boldsymbol{\beta}}}+\boldsymbol{\Lambda}_{P}^{T} \tilde{\boldsymbol{C}}_{\tilde{\boldsymbol{\beta}}}+\boldsymbol{\Lambda}_{L}^{T} \tilde{\boldsymbol{E}}_{\tilde{\boldsymbol{\beta}}}
$$

if $\boldsymbol{\Lambda}=\left[\boldsymbol{\Lambda}_{P}^{T} \boldsymbol{\Lambda}_{L}^{T}\right]^{T}$ satisfies the following derived adjoint equation:

$$
\left[\begin{array}{cc}
\tilde{\boldsymbol{C}}_{\tilde{\boldsymbol{P}}}^{T} & \mathbf{0} \\
\mathbf{0} & \tilde{\boldsymbol{E}}_{\tilde{\mathbf{L}}}^{T}
\end{array}\right]\left[\begin{array}{c}
\boldsymbol{\Lambda}_{P} \\
\boldsymbol{\Lambda}_{L}
\end{array}\right]=\left[\begin{array}{l}
-\mathscr{J}_{\tilde{\tilde{P}}}^{T} \\
-\mathscr{J}_{\tilde{\mathbf{L}}}^{T}
\end{array}\right],
$$

where in this context $\Lambda$ is termed as the vector of adjoint variables. The sparse structure of the matrix components

$$
\tilde{\boldsymbol{C}}_{\tilde{\boldsymbol{P}}}=\left[\begin{array}{cccccc}
\mathbf{I} & \mathbf{0} & \mathbf{0} & \cdots & \mathbf{0} & \mathbf{0} \\
-\mathbf{A}_{1} & \mathbf{I} & \mathbf{0} & \cdots & \mathbf{0} & \mathbf{0} \\
\mathbf{0} & -\mathbf{A}_{2} & \mathbf{I} & \cdots & \mathbf{0} & \mathbf{0} \\
\vdots & \vdots & \ddots & \ddots & \vdots & \vdots \\
\mathbf{0} & \mathbf{0} & \cdots & -\mathbf{A}_{N_{p}-2} & \mathbf{I} & 0 \\
\mathbf{0} & \mathbf{0} & \cdots & \mathbf{0} & -\mathbf{A}_{N_{p}-1} & \mathbf{I}
\end{array}\right],
$$

$$
\begin{gathered}
\tilde{\boldsymbol{E}}_{\tilde{\boldsymbol{L}}}=\left[\begin{array}{cccccc}
\mathbf{0} & \mathbf{0} & \mathbf{0} & \cdots & \mathbf{0} & \mathbf{0} \\
\mathbf{I} & \mathbf{0} & \mathbf{0} & \cdots & \mathbf{0} & \mathbf{0} \\
\mathbf{0} & \mathbf{I} & \mathbf{0} & \cdots & \mathbf{0} & \mathbf{0} \\
\vdots & \vdots & \ddots & \ddots & \vdots & \vdots \\
\mathbf{0} & \mathbf{0} & \cdots & \mathbf{I} & \mathbf{0} & \mathbf{0} \\
\mathbf{0} & \mathbf{0} & \cdots & \mathbf{0} & \mathbf{I} & \mathbf{0}
\end{array}\right], \\
\tilde{\boldsymbol{C}}_{\tilde{\boldsymbol{\beta}}}=\left[\begin{array}{cccccc}
\mathbf{0} & \mathbf{0} & \cdots & \mathbf{0} & \mathbf{0} \\
\left(\boldsymbol{C}_{2}\right)_{\boldsymbol{\beta}_{1}} & \mathbf{0} & \cdots & \mathbf{0} & \mathbf{0} \\
\mathbf{0} & \left(\boldsymbol{C}_{3}\right)_{\boldsymbol{\beta}_{2}} & \cdots & & \mathbf{0} & \mathbf{0} \\
\vdots & \vdots & \ddots & & \ddots & \vdots \\
\mathbf{0} & \mathbf{0} & \cdots & \left(\boldsymbol{C}_{N_{p}}\right)_{\boldsymbol{\beta}_{N_{p}-1}} & \mathbf{0}
\end{array}\right],
\end{gathered}
$$


with the following diagonal components:

$$
\begin{aligned}
\left(\boldsymbol{C}_{k}\right)_{\beta_{i, k-1}} & =-\frac{1-2 \beta_{i, k-1}}{1+\beta_{i, k-1}} B_{i, k-1}, \\
\left(\boldsymbol{E}_{k}\right)_{\beta_{i, k-1}} & =-\frac{1-\beta_{i, k-1}}{1+\beta_{i, k-1}} D_{i, k-1}
\end{aligned}
$$

allows us to derive the backward time-propagation of the adjoint variable (25)-(26). Consequently, the adjoint-based gradient (27) can be obtained by substituting (41)-(44) into the adjoint equation (40) and the MPC gradient expression (39). Note that $B_{i, k-1}$ and $D_{i, k-1}$ stand for control distribution components introduced in (14) and (15), respectively.

\begin{tabular}{ll} 
& \multicolumn{1}{c}{ ABBREVIATIONS } \\
ADM & actuator disc model \\
AGC & automatic generation control \\
AMPC & adjoint-based model predictive control \\
APC & active power control \\
CLD & coordinated load distribution \\
DEL & damage equivalent load \\
LES & large-eddy simulation \\
LQG & linear quadratic Gaussian \\
NBL & neutral boundary layer \\
PALM & PArallelized Large-eddy simulation Model \\
PI & proportional-integral \\
RMS & root mean square \\
STD & standard deviation \\
TSO & transmission system operator \\
WF & wind farm \\
WT & wind turbine
\end{tabular}

\section{ACKNOWLEDGMENTS}

Thanks are given to Gerald Steinfeld for his kind support on the PALM simulation code and LES analyses.

\section{REFERENCES}

[1] R. J. Barthelmie, K. Hansen, S. T. Frandsen, O. Rathmann, J. G. Schepers, W. Schlez, J. Phillips, K. Rados, A. Zervos, E. S. Politis, and P. K. Chaviaropoulos, "Modelling and measuring flow and wind turbine wakes in large wind farms offshore," Wind Energy, vol. 12, no. 5, pp. 431-444, 2009.

[2] A. Crespo, J. Hernández, and S. Frandsen, "Survey of modelling methods for wind turbine wakes and wind farms," Wind Energy, vol. 2, no. 1, pp. 1-24, 1999.

[3] F. Porté-Agel, M. Bastankhah, and S. Shamsoddin, "Wind-turbine and wind-farm flows: A review," Boundary-Layer Meteorology, vol. 174, no. 1, pp. 1-59, 2020.

[4] T. Knudsen, T. Bak, and M. Svenstrup, "Survey of wind farm control: power and fatigue optimization," Wind Energy, vol. 18, no. 8, pp. 1333-1351, 2015.

[5] J. R. Marden, S. D. Ruben, and L. Y. Pao, "A model-free approach to wind farm control using game theoretic methods," IEEE Transactions on Control Systems Technology, vol. 21, no. 4, pp. 1207-1214, 2013.

[6] J. Annoni, P. M. O. Gebraad, A. K. Scholbrock, P. A. Fleming, and J.-W. van Wingerden, "Analysis of axial-induction-based wind plant control using an engineering and a high-order wind plant model," Wind Energy, vol. 19, no. 6, pp. 1135-1150, 2016.

[7] P. M. O. Gebraad, F. W. Teeuwisse, J.-W. van Wingerden, P. A. Fleming, S. D. Ruben, J. R. Marden, and L. Y. Pao, "Wind plant power optimization through yaw control using a parametric model for wake effects: a CFD simulation study," Wind Energy, vol. 19, no. 1, pp. 95-114, 2016.
[8] P. A. Fleming, J. Aho, P. Gebraad, L. Y. Pao, and Y. Zhang, "Computational fluid dynamics simulation study of active power control in wind plants," in American Control Conference, 2016, pp. 1413-1420.

[9] J.-W. van Wingerden, L. Pao, J. Aho, and P. Fleming, "Active power control of waked wind farms." IFAC-PapersOnLine, vol. 50, no. 1, pp. 4484-4491, 2017.

[10] M. Steinbuch, W. de Boer, O. Bosgra, S. Peters, and J. Ploeg, "Optimal control of wind power plants," Journal of Wind Engineering and Industrial Aerodynamics, vol. 27, no. 1, pp. 237-246, 1988.

[11] M. Soleimanzadeh, R. Wisniewski, and K. Johnson, "A distributed optimization framework for wind farms," Journal of Wind Engineering and Industrial Aerodynamics, vol. 123, pp. 88-98, 2013.

[12] V. Spudić, C. Conte, M. Baotić, and M. Morari, "Cooperative distributed model predictive control for wind farms," Optimal Control Applications and Methods, vol. 36, no. 3, pp. 333-352, 2015.

[13] A. D. Hansen, P. Sørensen, F. Iov, and F. Blaabjerg, "Centralised power control of wind farm with doubly fed induction generators," Renewable Energy, vol. 31, no. 7, pp. 935-951, 2006.

[14] I. M. de Alegria, J. Andreu, J. L. Martin, P. Ibanez, J. L. Villate, and H. Camblong, "Connection requirements for wind farms: A survey on technical requierements and regulation," Renewable and Sustainable Energy Reviews, vol. 11, no. 8, pp. 1858-1872, 2007.

[15] J. Aho, A. Buckspan, J. Laks, P. A. Fleming, Y. Jeong, F. Dunne, M. Churchfield, L. Y. Pao, and K. Johnson, "A tutorial of wind turbine control for supporting grid frequency through active power control," in American Control Conference, 2012, pp. 3120-3131.

[16] J. Aho, P. A. Fleming, and L. Y. Pao, "Active power control of wind turbines for ancillary services: A comparison of pitch and torque control methodologies," in American Control Conference, 2016, pp. 1407-1412.

[17] M. Vali, V. Petrović, G. Steinfeld, L. Y. Pao, and M. Kühn, "An active power control approach for wake-induced load alleviation in a fully developed wind farm boundary layer," Wind Energy Science, vol. 4, no. 1, pp. 139-161, 2019.

[18] J. P. Goit and J. Meyers, "Optimal control of energy extraction in wind-farm boundary layers," Journal of Fluid Mechanics, vol. 768, pp. 5-50, 2015.

[19] W. Munters and J. Meyers, "Dynamic strategies for yaw and induction control of wind farms based on large-eddy simulation and optimization," Energies, vol. 11, no. 1, 2018.

[20] M. Vali, V. Petrović, S. Boersma, J.-W. van Wingerden, L. Y. Pao, and M. Kühn, "Adjoint-based model predictive control for optimal energy extraction in waked wind farms," Control Engineering Practice, vol. 84, pp. $48-62,2019$.

[21] C. R. Shapiro, P. Bauweraerts, J. Meyers, C. Meneveau, and D. F. Gayme, "Model-based receding horizon control of wind farms for secondary frequency regulation," Wind Energy, vol. 20, no. 7, pp. 1261-1275, 2017.

[22] M. Vali, V. Petrović, S. Boersma, J.-W. van Wingerden, L. Y. Pao, and M. Kühn, "Model predictive active power control of waked wind farms," in American Control Conference, 2018, pp. 707-714.

[23] S. Boersma, B. Doekemeijer, S. Siniscalchi-Minna, and J.-W. van Wingerden, "A constrained wind farm controller providing secondary frequency regulation: An LES study," Renewable Energy, vol. 134, pp. 639-652, 2019.

[24] A. E. Yilmaz, "LES-based optimal flow control with applications to wind turbines," Ph.D. dissertation, Catholic University of Leuven, Belgium, 2019.

[25] B. L. Jones, P. Heins, E. Kerrigan, J. Morrison, and A. Sharma, "Modelling for robust feedback control of fluid flows," Journal of Fluid Mechanics, vol. 769, p. 687-722, 2015.

[26] M. Soleimanzadeh, R. Wisniewski, and A. Brand, "State-space representation of the wind flow model in wind farms," Wind Energy, vol. 17, no. 4, pp. 627-639, 2014.

[27] J. Annoni and P. Seiler, "A low-order model for wind farm control," in American Control Conference, 2015, pp. 1721-1727.

[28] A. Rott, S. Boersma, J.-W. van Wingerden, and M. Kühn, "Dynamic flow model for real-time application in wind farm control," Journal of Physics: Conference Series, vol. 854, no. 1, p. 012039, 2017.

[29] S. Boersma, B. Doekemeijer, M. Vali, J. Meyers, and J.-W. van Wingerden, "A control-oriented dynamic wind farm model: WFSim," Wind Energy Science, vol. 3, no. 1, pp. 75-95, 2018.

[30] S. Boersma, M. Vali, M. Kühn, and J.-W. van Wingerden, "Quasi linear parameter varying modeling for wind farm control using the 
2D Navier-Stokes equations," in American Control Conference, 2016 pp. 4409-4414.

[31] B. M. Doekemeijer, S. Boersma, L. Y. Pao, T. Knudsen, and J.-W. van Wingerden, "Online model calibration for a simplified LES model in pursuit of real-time closed-loop wind farm control," Wind Energy Science, vol. 3, no. 2, pp. 749-765, 2018.

[32] C. J. Bay, J. Annoni, T. Taylor, L. Pao, and K. Johnson, "Active power control for wind farms using distributed model predictive control and nearest neighbor communication," in American Control Conference, 2018, pp. 682-687.

[33] V. Petrović, J. Schottler, I. Neunaber, M. Hölling, and M. Kühn, "Wind tunnel validation of a closed loop active power control for wind farms," Journal of Physics: Conference Series, vol. 1037, no. 3, p. $032020,2018$.

[34] D. Madjidian, "Scalable minimum fatigue control of dispatchable wind farms," Wind Energy, vol. 19, no. 10, pp. 1933-1944, 2016.

[35] J. Kazda and N. A. Cutululis, "Model-optimized dispatch for closedloop power control of waked wind farms," IEEE Transactions on Control Systems Technology, pp. 1-8, 2019.

[36] M. Bromm, A. Rott, H. Beck, L. Vollmer, G. Steinfeld, and M. Kühn, "Field investigation on the influence of yaw misalignment on the propagation of wind turbine wakes," Wind Energy, vol. 21, no. 11, pp. 1011-1028, 2018.

[37] M. Vali, V. Petrović, L. Y. Pao, and M. Kühn, "Lifetime extension of waked wind farms using active power control," Journal of Physics: Conference Series, vol. 1256, p. 012029, 2019.

[38] J. Meyers and C. Meneveau, "Large eddy simulations of large wind-turbine arrays in the atmospheric boundary layer," 48th AIAA Aerospace Sciences Meeting Including the New Horizons Forum and Aerospace Exposition, 2010.

[39] F. Porté-Agel, Y.-T. Wu, H. Lu, and R. J. Conzemius, "Large eddy simulation of atmospheric boundary layer flow through wind turbines and wind farms," Journal of Wind Engineering and Industrial Aerodynamics, vol. 99, no. 4, pp. 154-168, 2011.

[40] B. Sanderse, "Aerodynamics of wind turbine wakes," Technical report ECN-E-09-016, pp. Energy Research Center of the Netherlands (ECN), the Netherlands, 2009.

[41] B. Maronga, M. Gryschka, R. Heinze, F. Hoffmann, F. KananiSühring, M. Keck, K. Ketelsen, M. O. Letzel, M. Sühring, and S. Raasch, "The Parallelized Large-Eddy Simulation Model (PALM) version 4.0 for atmospheric and oceanic flows: model formulation, recent developments, and future perspectives," Geoscientific Model Development, vol. 8, no. 8, pp. 2515-2551, 2015.

[42] B. Witha, G. Steinfeld, M. Dörenkämper, and D. Heinemann, "Largeeddy simulation of multiple wakes in offshore wind farms," Journal of Physics: Conference Series, vol. 555, no. 1, p. 012108, 2014.

[43] U. Schumann, "Subgrid scale model for finite difference simulations of turbulent flows in plane channels and annuli," Journal of Computational Physics, vol. 18, no. 4, pp. 376-404, 1975.

[44] D. Schlipf, D. J. Schlipf, and M. Kühn, "Nonlinear model predictive control of wind turbines using LIDAR," Wind Energy, vol. 16, no. 7, pp. 1107-1129, 2013

[45] R. Gasch and J. Twele, Wind power plants: fundamentals, design, construction and operation, Second edition. Springer Science \& Business Media, 2011.

[46] M. O. Hansen, Aerodynamics of wind turbines, Second edition. Earthscan, 2008.

[47] E. Bossanyi, B. Savini, M. Iribas, M. Hau, B. Fischer, D. Schlipf, T. van Engelen, M. Rossetti, and C. E. Carcangiu, "Advanced controller research for multi-MW wind turbines in the UPWIND project," Wind Energy, vol. 15, no. 1, pp. 119-145, 2012.

[48] S. Qin and T. A. Badgwell, "A survey of industrial model predictive control technology," Control Engineering Practice, vol. 11, no. 7, pp. 733-764, 2003.

[49] J. M. Jonkman and M. L. Buhl, "FAST manual user's guide," NREL report No. NREL/EL-500-38230, Boulder, USA, 2005.

[50] C. Pilong, "PJM manual 12: Balancing operations," 2013, 30th ed., PJM.

[51] IEC, "International electrotechnical commission, wind turbines-part 1: Design requirements," IEC 61400-1:2005(E), Third edition, 2005.

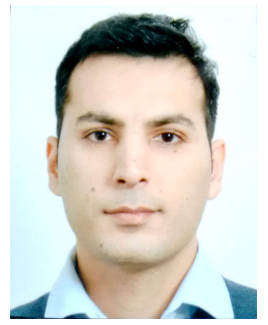

Mehdi Vali received his M.Sc. degree in information and automation engineering from the University of Bremen, Germany, in 2015, and his Ph.D. degree focusing on wind energy control systems from the University of Oldenburg, Germany, in 2019. He is currently a postdoctoral researcher at the University of Oldenburg, Germany. His research interests include optimal control and multivariable control systems with application to wind farm control and wind turbine control.

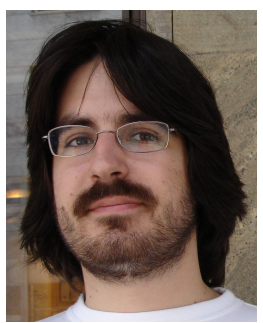

Vlaho Petrović received his Ph.D. from the University of Zagreb in 2013. Since 2016, he has been working as a senior researcher at the University of Oldenburg. His research interests include wind turbine and wind farm control with the focus on optimal and predictive control, as well as experimental validation of control algorithms in wind tunnel and field experiments.

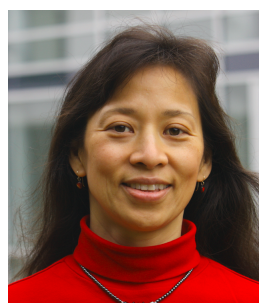

Lucy Y. Pao is a Palmer Endowed Chair Professor in the Electrical, Computer, and Energy Engineering Department at the University of Colorado Boulder. She earned B.S., M.S., and Ph.D. degrees in Electrical Engineering from Stanford University. Her research has primarily focused on engineering control systems, with applications ranging from atomic force microscopes to multimegawatt wind energy systems. She is a Fellow of the IEEE and IFAC. Selected recent awards include the 2015 SIAM Journal on Control and Optimization Best Paper Prize, the 2017 AACC Control Engineering Practice Award, the 2017 European Academy of Wind Energy Scientific Award, and the 2019 ASME Nyquist Lecturer Award. Selected recent and current professional society activities include being a Fellow of the Renewable and Sustainable Energy Institute (2009- ), a member of the IFAC Executive Board (2017-2020), the Education Liaison on the IFAC Technical Board (2020-2023), and the IFAC Pavel J. Nowacki Distinguished Lecturer (2020-2023).

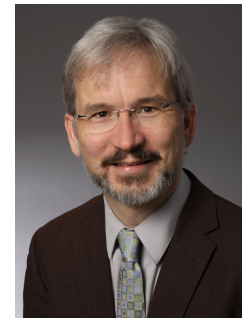

Martin Kühn studied Mechanical Engineering and Physical Engineering at Hanover, Berlin, both Germany and Delft, the Netherlands. In the 1990s he worked as a researcher at TU Delft where he completed a ground-breaking $\mathrm{PhD}$ on dynamics and design optimisation of offshore wind turbines. During five years affiliated with an multinational $\mathrm{OEM}$, he acted as engineering project manager for two of the early offshore wind farms in Sweden and Ireland. Since 2004 he is full professor of wind energy systems, first Stuttgart University and currently ForWind - Center for Wind Energy Research, Carl von Ossietzky University Oldenburg, both Germany. His research interests cover control and load monitoring of wind turbines and wind farms, in-field measurement techniques incl. lidar sensing of wind farm flow, dynamics and design of wind energy systems. 